\title{
Detection and Discrimination of First- and Second-Order Motion in Patients with Unilateral Brain Damage
}

\author{
Mark W. Greenlee ${ }^{1}$ and Andy T. Smith ${ }^{2}$ \\ ${ }^{1}$ Neurologische Universitätsklinik, Freiburg, Germany, and 2Department of Psychology, Royal Holloway College, University \\ of London, Egham, Surrey, United Kingdom
}

The present investigation explored the extent to which extrastriate cortex is necessary for various aspects of motion processing and whether the processing of first-order (Fourier) and second-order (non-Fourier) motion involves the same extrastriate cortical regions. Orientation, direction, and speed discrimination thresholds were measured in 21 patients with unilateral damage to the lateral occipital, temporal, or posterior parietal cortex. Their results were compared with those of 14 agematched control subjects. The stimuli were static random-dot noise patterns, the luminance of which (first-order) or contrast (second-order) was modulated by a drifting sinusoid. Each image was presented at an eccentricity of $5.6 \mathrm{deg}$ in one of the four visual quadrants. The contrasts required to identify orientation and direction were measured in a forced-choice paradigm for three speeds $(1.5,3$, and $6 \mathrm{deg} / \mathrm{sec})$. Speed discrimination performance was measured for stimuli presented simultaneously in two of the four quadrants. The results indicate the following: (1) orientation thresholds were increased only slightly in the patients; (2) direction thresholds were modestly elevated, and this effect was more pronounced for second-order stimuli than for first-order stimuli; (3) speed discrimination thresholds were elevated significantly in the patients with lesions in the region bordering superior-temporal and lateral-occipital cortex; and (4) speed discrimination thresholds for first-order stimuli were more elevated than those for second-order stimuli. The results suggest that there is substantial overlap in the cortical areas involved in first- and second-order speed discrimination.

Key words: motion perception; direction discrimination; speed discrimination; second-order motion; human cortex; contrast sensitivity; spatiotemporal vision; psychophysics
There is mounting evidence that the primate brain contains extrastriate areas specialized for the processing of stimulus motion (Maunsell and Van Essen, 1983; Albright, 1984; Mikami et al., 1986a,b; Movshon et al., 1986; Newsome et al., 1986; Rodman and Albright, 1987; Snowden et al., 1992). Motion signals not only are carried by variations in luminance or color (the first-order characteristics of the image) but also can be carried by differences in second-order image characteristics such as texture, disparity, and contrast (Smith, 1994). Derrington and Badcock (1985) argued that moving contrast modulations are detected by a different mechanism from that which detects luminance-defined (firstorder) motion. The idea of two separate motion detection pathways has since been incorporated into computational models (Chubb and Sperling, 1988; Wilson et al., 1992). In these models, the two motion detection systems are both low level mechanisms, and they operate in parallel. One (first-order) is modeled with the principle of motion energy detection (Adelson and Bergen, 1985). In the other (second-order), the luminance signal first is filtered and then is rectified or squared before being passed to a motion energy detection stage. The nonlinear transformation has the

\footnotetext{
Received July 15, 1996; revised Oct. 25, 1996; accepted Nov. 1, 1996.

This research was supported by the Deutsche Forschungsgemeinschaft (Gr 988/10) and the European Community (Human Capital and Mobility Program). M.W.G. is currently supported by the Hermann and Lilly Schilling Foundation. We express our appreciation to the persons who served as subjects in these experiments and to Professors W. Seeger and C. H. Lücking for their helpful comments.

Correspondence should be addressed to Dr. Mark W. Greenlee, Neurologische Universitätsklinik, University of Freiburg, Breisacherstr. 64, 79106 Freiburg, Germany.

Copyright (C) 1997 Society for Neuroscience $\quad 0270-6474 / 97 / 170804-15 \$ 05.00 / 0$
}

effect of introducing first-order motion, which is correlated with the second-order motion in the original image.

Several motion detection mechanisms may exist in the human visual system, and these mechanisms might be located in different areas of cerebral cortex. The human homolog of V5/MT has been identified via functional imaging techniques (Zeki et al., 1991; Watson et al., 1993; Tootell et al., 1995). Among studies of the effects of brain lesions, severe impairments in motion perception have been reported in only one case with bilateral damage in posterior lateral cortex (Zihl et al., 1983, 1991), but the extensive nature of the damage does not allow precise localization of the motion areas. However, Plant and colleagues (Plant and $\mathrm{Na}$ kayama, 1993; Plant et al., 1993) reported results from three patients who showed marked impairments in direction and speed discrimination. These patients had less difficulty discriminating the spatial frequency of drifting gratings, suggesting a motionspecific deficit. Vaina and coworkers $(1989,1994)$ have explored the ability of individual patients to integrate direction information in global dot motion. In a recent study, Greenlee et al. (1995) found that patients with unilateral cortical damage in the temporal-occipital-parietal border region exhibited significant impairments in their ability to discriminate the speeds of sequentially presented first-order motion stimuli. Thus, little is known concerning which anatomical regions of the human brain mediate second-order motion perception.

We have explored sensitivity to the orientation, direction, and speed of first- and second-order motion stimuli in patients with unilateral damage to the temporal, lateral occipital, and posterior parietal cortex. The results indicate that damage to posterior superior temporal cortex or inferior parietal cortex significantly 
impairs the discrimination of the speed of suprathreshold firstorder and, to a lesser extent, second-order stimuli. Orientation and direction thresholds are less affected.

\section{MATERIALS AND METHODS}

Patients and control subjects. The observers were 21 former neurological patients who showed objective signs of focal cortical lesion in one of the cerebral hemispheres. Table 1 presents the relevant clinical data on the patient sample. The patients were selected from the medical archives of the Department of Neurosurgery of the University of Freiburg. Twenty patients had undergone surgical resection of a vessel malformation or a tumor, the malignancy of which did not exceed WHO II (2 astrocytomas, 2 meningioma, 5 arteriovenous malformations, and 11 cavernous angiomas). One patient (PAT08) had a well defined lesion resulting from ischemic infarction. All lesions were located primarily in the cortical gray matter but inevitably included white matter in some cases. The patients were studied, on average, 34.5 months after surgery (the range was 0-94 months). They were recruited with informed consent after consulting their general practitioner or neurologist. During the selection process, we excluded any patients fulfilling any of the following criteria: age $>70$ years, more than one cerebral lesion, glioblastoma, or metastases, an ill-defined lesion (e.g., edema), signs of visual neglect in the case history, pronounced neuropsychological disorders, radiation therapy, on-going high-dose anticonvulsant therapy with potentially sedating drugs, and/or drug intoxication. The patient group consisted of 11 females and 10 males. Three of the patients (see Table 1) were left-handed. Twelve patients had damage in the left cerebral hemisphere, and nine had a lesion in the right hemisphere (Table 1). The lesion in 11 patients was located in, or extended into, the border region of the superior temporal and occipital cortex, referred to in the following as the superior temporal (ST) group. These patients form our region-of-interest group. In addition, four patients showed damage in the lateral parietal cortex dorsal to and not including the angular gyrus (referred as the LIP group), and six patients had a lesion located in the inferior temporal cortex (referred to as the IT group). Ten of the patients participated in an earlier investigation on the discrimination and retention of the speed of drifting gratings (Greenlee et al., 1995). Eleven patients were receiving antiepileptic therapy. The type and daily dosage levels of the anticonvulsants used are given in Table 1.

The 14 control subjects were approximately matched for age, sex, and handedness. The mean age of the patients was 40.6 years, $\mathrm{SD}=9.5$ years (range, 26-56 years), and that of the control group was 35.2 years, $\mathrm{SD}=$ 9.5 years (range, 21-53 years). There was no significant difference between the mean ages of the patient and control groups $\left(F_{1,33}=2.7, p>\right.$ $0.1)$, nor was there a difference between the mean age of the different patient groups $\left(F_{2,18}=1.6, p>0.2\right)$.

Analysis of lesioned cortical area. The location and extent of the lesioned cortical area were determined using pre- and postoperative computed tomograms and magnetic resonance images as well as the protocol from surgery. An outline of the lesioned area was transferred onto standardized templates derived from a computed tomographic atlas (Seeger, 1978; Nadjmi et al., 1991). Then the templates were stacked appropriately to yield a pseudo-three-dimensional representation. These reconstructions are shown for each patient in Figure 1, the darkly shaded areas depicting the location and extent of the cortical lesion.

Stimuli. The stimuli were generated by a Matrox image processing system and were displayed on a monochrome monitor with P4 (white) phosphor. Each stimulus consisted of a $5 \mathrm{deg}$ square grating patch. The spatial frequency of the grating was always 1 cycle/degree (c/deg). The orientation of the grating could be either horizontal or vertical, and it could drift in either direction along the axis orthogonal to its orientation. The gratings could be either first-order (luminance-defined) or secondorder (contrast-defined).

Second-order gratings consisted of static, high-pass-filtered twodimensional noise (referred to as the carrier), the contrast of which was modulated sinusoidally in one dimension. They were constructed as follows. First, a sample of binary two-dimensional noise was drawn (i.e. each pixel was assigned one of two values, light or dark, at random). The noise had a pixel size of $2 \mathrm{~min}$ arc. Then the noise was spatially filtered with conventional Fourier techniques. An ideal high-pass filter with a cut-off at $1 \mathrm{c} / \mathrm{deg}$ was used to remove all spatial frequencies below that value. The purpose of the filtering was to remove local first-order artifacts that arise when unfiltered noise carriers are used (Smith and Ledgeway, 1996). The filtered carrier was constructed off-line and stored on disk. To generate second-order motion, we loaded the filtered carrier into one frame buffer, and drew a sine grating in another. Then the two images were multiplied together. For the purpose of multiplication, the carrier was treated as signed, and the sine grating was treated as unsigned (raised). This produced a sinusoidal modulation of the contrast of the carrier, the spatial frequency of which was the same as that of the modulating waveform (1 c/deg). The image had the appearance of a grating defined by contrast (see Fig. 2). To produce motion, we repeatedly incremented the phase of the multiplying sinusoid by a small constant amount at a rate of $67 \mathrm{~Hz}$. The carrier remained stationary, and the multiplication was repeated for each frame. The multiplication was performed in real time with a look-up table. This gave smooth motion of the contrast-defined grating at a constant velocity (determined by the size of the phase shift) while the noise itself remained stationary. The mean (space-averaged) contrast of the carrier was always $50 \%$. The modulation depth varied among experimental conditions.

First-order gratings were produced in the same way except that the drifting sine grating was added to, rather than multiplied by, the static high-pass-filtered carrier. Again, this operation was performed in real time, and the phase of the grating was updated at $67 \mathrm{~Hz}$. The resulting image had the appearance of a conventional sine grating drifting smoothly across stationary noise. The mean contrast of the noise again was fixed at $50 \%$, and the contrast of the drifting grating varied among experimental conditions. The noise was included to make the first-order images as similar in appearance as possible to the second-order images. The presence of stationary noise could have an influence on perceived speed (although we have results suggesting that this is not the case in healthy observers), and so it was important that both types of image were affected equally. Perhaps more importantly, it is possible that patients with cortical lesions might have difficulties in judging motion in the presence of stationary noise, which could lead to selective second-order deficits of an artifactual nature if noise also were not added to the first-order stimuli. In all cases, the noise patches were reported to be clearly visible by the patients and controls at the viewing distance used.

A central fixation point was always provided. Images of the type described above could be presented in any of the four visual quadrants. The center of each $5 \mathrm{deg}$ square image was located at an eccentricity of $5.6 \mathrm{deg}$, leaving a $3 \mathrm{deg}$ gap between adjacent images when more than one was presented (see Fig. 2). The remainder of the display was filled with a uniform gray of the same luminance $\left(20 \mathrm{~cd} \mathrm{~m}^{-2}\right)$ as the stimuli. The duration of each stimulus was $0.5 \mathrm{sec}$. Stimulus onset and offset were abrupt.

Procedure. Two types of measurement were conducted. In both experiments, the observers viewed the display binocularly from a distance of $0.57 \mathrm{~m}$. Constant distance and head orientation were maintained by having the observer rest the back of his/her head on a headrest. The observers were instructed to fixate the central fixation point, which was displayed on the monitor throughout the experiment.

Orientation/direction identification thresholds. The contrast (first-order motion) or contrast modulation depth (second-order motion) needed to identify the orientation and the direction of motion of a stimulus presented in one quadrant were determined by using a forced choice procedure. Each trial was announced by a computer-generated auditory tone. Within each trial, four $5 \mathrm{deg}$ square patches of stationary, filtered, two-dimensional noise were presented, one in each of the four visual quadrants. In one of the four patches, chosen at random, either the luminance (first-order motion conditions) or the contrast (second-order motion conditions) of the random dot background was modulated by a one-dimensional sine function as described above. In two binary independent judgments, the observers signaled (1) whether the grating was vertical or horizontal and (2) whether the perceived direction corresponded to one (left, up) or the other (right, down) class of direction. Responses were made verbally and were keyed into the computer by the experimenter. Orientation and direction judgments were scored independently. The screen was blank (except for the fixation spot) for at least 3 sec between trials.

Orientation and direction thresholds were measured by the method of constant stimuli. Each run consisted of 120 trials. The location of the stimulus varied randomly from trial to trial, each quadrant being presented 30 times in total. The 30 trials in each quadrant consisted of five trials at each of six contrasts (modulation depths) of the sine grating, carrier contrast remaining fixed at $50 \%$. The six modulation depths were chosen on the basis of pilot studies to span the threshold. Thresholds were obtained for three speeds $(1.5,3$, and $6 \mathrm{deg} / \mathrm{sec})$ in separate runs for each image type (first-order and second-order), making six runs in total 
Table 1. Clinical data on the 21 patients who participated in the study

\begin{tabular}{|c|c|c|c|c|c|c|c|}
\hline Patient & $\begin{array}{l}\text { Lesioned } \\
\text { side }\end{array}$ & $\begin{array}{l}\text { Lesion } \\
\text { location }\end{array}$ & $\begin{array}{l}\text { Age } \\
\text { (years) }\end{array}$ & Sex & Diagnosis & Handedness & Date of surgery \\
\hline PAT01 & left & ST & 40 & male & arteriovenous malformation & left & 01.05 .1991 \\
\hline PAT02 & left & ST & 52 & male & cavernous angioma & right & 01.11.1990 \\
\hline PAT03 & left & ST & 53 & female & cavernous angioma & right & 01.08 .1990 \\
\hline PAT04 & left & ST & 37 & female & cavernous angioma & right & 17.10.1989 \\
\hline PAT05 & left & ST & 42 & male & cavernous angioma & left & 24.05.1992 \\
\hline PAT06 & left & ST & 45 & female & cavernous angioma & right & 25.04.1994 \\
\hline PAT07 & left & ST & 45 & female & cavernous angioma & right & 28.07.1994 \\
\hline PAT08 & right & ST & 54 & female & ischemic infarction & right & $\bullet$ \\
\hline PAT09 & right & ST & 54 & male & cavernous angioma & right & 09.11.1993 \\
\hline PAT10 & right & ST & 27 & male & arteriovenous malformation & right & 05.10 .1993 \\
\hline PAT11 & right & ST & 35 & male & arteriovenous malformation & right & 01.12.1995 \\
\hline PAT12 & left & LIP & 32 & male & arteriovenous malformation & right & 13.03.1991 \\
\hline PAT13 & left & LIP & 31 & female & arteriovenous malformation & right & 06.12.1991 \\
\hline PAT14 & right & LIP & 32 & male & cavernous angioma & left & 01.05 .1993 \\
\hline PAT15 & right & LIP & 56 & female & meningioma & right & 05.02.1989 \\
\hline PAT16 & left & IT & 26 & female & cavernous angioma & right & 01.05.1993 \\
\hline PAT17 & left & IT & 33 & female & astrocytoma & right & 03.01.1992 \\
\hline PAT18 & left & IT & 46 & male & cavernous angioma & right & 15.02.1995 \\
\hline PAT19 & right & IT & 30 & male & meningioma & right & 01.02.1991 \\
\hline PAT20 & right & IT & 41 & female & cavernous angioma & right & 01.11.1986 \\
\hline PAT21 & right & IT & 42 & female & astrocytoma & right & 07.05.1990 \\
\hline
\end{tabular}

GM, Grand mal seizure; ST, superior temporal; IT, inferotemporal; LIP, lateral inferoparietal.

per subject. For each visual quadrant and speed, a Weibull function was fit to the psychometric function (percentage of correct responses as a function of modulation depth) with an iterative algorithm suggested by Foster and Bischof (1991). The 75\% correct point on this function was taken as the threshold value. Separate curves were fit to the data for orientation and direction to give two independent threshold values. The Foster and Bischof algorithm provided an estimate of the slope and the goodness of fit. These values were analyzed for data aggregated over visual quadrants (i.e., 120 trials per psychometric function) to control for the quality of the curve fit. In cases where the algorithm could not provide a good fit to the data, a least-squares fit was made to the data via a Weibull function with an average slope.

Speed discrimination thresholds. In a second experiment, speed discrimination thresholds were determined for patterns shown at a constant suprathreshold contrast level. Speed discrimination thresholds are defined as the minimum detectable difference in the speeds of two otherwise-identical motion stimuli. Within each trial, four 5 deg square patches of stationary, filtered two-dimensional noise again were presented, one in each of the four visual quadrants. On each trial, two motion stimuli were presented simultaneously in different quadrants, and the other two quadrants remained unmodulated. One of the motion stimuli (the reference stimulus) had a drift speed chosen randomly from three speeds: $2.7,3.0$, and $3.3 \mathrm{deg} / \mathrm{sec}$. The purpose of this random speed jitter was to eliminate the possibility that the subject could learn to identify a single reference speed. The other motion stimulus (the test stimulus) had a higher speed, which differed from that of the reference by $\Delta S$. Reference and test stimuli always drifted in the same direction. The task of the subject was to say which of the two stimuli had the higher speed. The contrast of the noise was again $50 \%$. The contrast (modulation depth) of the grating was fixed at a constant suprathreshold level. In the case of second-order motion, the contrast modulation depth was $87 \%$. This ensured that the moving grating was easily visible for all patients. In the case of first-order motion, the contrast of the sine grating was chosen to have equal visibility (for a healthy observer) to the second-order stimuli. This level was $6 \%$ and was chosen as follows. Accurate orientation/direction thresholds were measured in pilot work for two healthy observers (one of the authors, A.S., and a naive subject, T.F.) who both were experienced observers. The second-order modulation depth used in the main experiment $(87 \%)$ was divided by the mean second-order direction threshold for the two healthy observers to determine the mul- tiple of threshold corresponding to $87 \%$. The first-order contrast for the speed discrimination experiment was chosen by multiplying the first-order direction threshold by the same factor. Thus, first-order and second-order stimuli were presented at the same multiple of direction identification threshold for a standard observer.

Speed discrimination thresholds were measured by the method of constant stimuli. Separate discrimination thresholds were obtained for pairs of stimuli presented in each of four pairs of quadrants: the two right-hand quadrants, the two left-hand quadrants, the two upper quadrants, and the two lower quadrants. For example, on a trial using the two upper quadrants, two motion stimuli appeared in the upper quadrants, one of which was the reference and the other the test, determined at random. Upper and lower hemifields were tested in a single run (i.e. trials using the two upper quadrants were interleaved randomly with those using the two lower quadrants). In this case the orientation of both gratings was vertical. Right and left quadrants were tested in a separate run, in which horizontally oriented gratings were used. Each of these runs was repeated twice, once using each of the two possible directions of motion, giving a total of four runs. First-order and second-order stimuli were tested in separate runs, making eight runs altogether per subject. Each run contained 120 trials. Six speed increments were used, chosen on the basis of pilot studies. Each of these was presented 10 times (total of 60 trials) in random order for each of the two hemifields used.

Discrimination thresholds were obtained for each subject in each condition by fitting Weibull curves to the psychometric function relating the percentage of correct responses to $\Delta S$. The threshold was taken as the value corresponding to the $75 \%$ performance level on the curve. Because no effects of drift direction were observed, the data for the two opposite drift directions were pooled when curves were fit to the data.

\section{RESULTS}

\section{Orientation/direction identification thresholds for first- and second-order stimuli}

The results of the orientation and direction threshold measurements are shown for the 21 patients $(a-c)$, and can be compared with the findings of the 14 controls $(d)$ in Figures 3 (first-order motion) and 4 (second-order motion). The logarithm of the 


\begin{tabular}{|c|c|c|c|}
\hline $\begin{array}{l}\text { Months } \\
\text { since } \\
\text { surgery }\end{array}$ & Symptoms before surgery & Symptoms at time of study & $\begin{array}{l}\text { Medication } \\
\text { (possibly sedative) } \\
\text { at time of study }\end{array}$ \\
\hline 37 & lower right quadrant defect for small targets & lower right quadrant defect for small targets & none \\
\hline 44 & $2 \times \mathrm{GM}$ & none & none \\
\hline 47 & GM, partial complex seizures, mild aphasia & none & phenytoin $500 \mathrm{mg}$ \\
\hline 57 & & & none \\
\hline 28 & $1 \times \mathrm{GM}$ & none & carbamazepine $400 \mathrm{mg}$ \\
\hline 17 & headache, impaired vigilance, short-term memory disorder & headache, short-term memory disorder & carbamazepine $1200 \mathrm{mg}$ \\
\hline 15 & generalized seizures & & carbamazepine $200 \mathrm{mg}$ \\
\hline$\bullet$ & none & none & none \\
\hline 10 & $1 \times \mathrm{GM}$ & none & carbamazepine $400 \mathrm{mg}$ \\
\hline 24 & several GM, lower left visual quadrant defect & left hemiparesis, lower left visual quadrant defect & phenytoin $800 \mathrm{mg}$ \\
\hline 0 & $1 \times \mathrm{GM}$ & none & carbamazepine $800 \mathrm{mg}$ \\
\hline 40 & $1 \times \mathrm{GM}$ & none & none \\
\hline 50 & mild aphasia, hemiparesis right & mild aphasia, right hemiparesis & carbamazepine $400 \mathrm{mg}$ \\
\hline 16 & $3 \times \mathrm{GM}$ & none & carbamazepine $400 \mathrm{mg}$ \\
\hline 80 & hypaesthesia in left lower limb & none & none \\
\hline 13 & severe headache & none & none \\
\hline 32 & partial seizures & none & none \\
\hline 8 & partial seizures, $1 \times \mathrm{GM}$ & none & carbamazepine $400 \mathrm{mg}$ \\
\hline 28 & lower left quadrant defect for small targets & lower lower quadrant defect for small targets & none \\
\hline 94 & several seizures & none & none \\
\hline 49 & $1 \times \mathrm{GM}$ & none & carbamazepine $900 \mathrm{mg}$ \\
\hline
\end{tabular}

threshold contrast is shown as a function of reference speed for each visual quadrant. The results for the 11 patients with damage in the superior temporal/occipital border region (the ST group) are presented in $a$, those for four patients with inferior parietal damage (the LIP group) in $b$, and the results for six patients with damage in the inferior temporal cortex (the IT group) in $c$. Open symbols signify thresholds for orientation, and filled symbols represent thresholds for direction of motion. Note that the data are collapsed across patients with left and right hemisphere damage, and the visual quadrants are identified with respect to the damaged hemisphere (i.e., ipsilesional or contralesional).

A five-way ANOVA was performed on the logarithm of the contrast threshold values, which tested the effects of the betweensubjects factor experimental group (i.e., patients vs controls), and the within-subjects factors stimulus dimension (orientation, direction), reference speed $(1.5,3$, and $6 \mathrm{deg} / \mathrm{sec})$, order of motion (first, second), and visual quadrant. Overall, the effect of the experiment group was highly significant $\left(F_{1,33}=9.58, p=0.004\right)$, which indicates that the patients' ability to discriminate the orientation and direction of the moving stimuli was significantly impaired, as compared with the controls. The main effects of stimulus dimension $\left(F_{1,33}=121.7, p=0.0001\right)$ and reference speed $\left(F_{2,66}=18.88, p=0.0001\right)$ were also highly significant. The effect of stimulus dimension indicates that the subjects had lower thresholds for detecting the orientation of the modulation, as compared with detecting the direction of the motion. Inspection of the data shows that this difference is largely attributable to the second-order motion condition. The effect of reference speed indicates the trend seen in Figures 3 and 4 that thresholds decreased with increasing stimulus speed. The main effect of motion type (first-, second-order) was also statistically significant. However, this effect is trivial, because first-order and second-order motion thresholds are measured on different scales (contrast and modulation depth, respectively). The effect of visual field was moderately significant $\left(F_{3,99}=3.27, p=0.024\right)$. The visual field effect is only consistent for the ST patients (Figs. 3a, 4a) and is most pronounced for first-order motion. A post hoc weighted means comparison of the log thresholds for ST patients indicated an effect of ipsilesional-contralesional visual field averaged over upper and lower quadrants $\left(F_{1,10}=4.27 ; p=0.048\right)$, indicating that thresholds for these patients tend to be higher in the contralesional visual field.

None of the interactions between the factor experimental group and the within-subjects factors was significant. This lack of interaction suggests that the stimulus variations (dimension judged, type of motion, location, speed) had similar effects in both patient and control groups. Among the within-subjects factors, the largest interaction occurred between the dimension judged and the type of motion $\left(F_{1,33}=115.1, p=0.0001\right)$ and, to a lesser extent, between dimension and reference speed $\left(F_{2,66}=6.44, p=0.003\right)$. These interaction terms suggest that the variations in motion type and speed affected the two types of threshold differently. As is evident in Figures 3 and 4, orientation and direction thresholds are approximately at the same level for first-order motion but diverge considerably for second-order motion. This is true for both patients and controls and is in line with our recent findings for healthy observers (Smith and Ledgeway, 1997). As discussed in that paper, the fact that direction and orientation thresholds are different for second-order motion indicates that the secondorder patterns were, for the most part, free of first-order artifacts. This finding suggests that our stimuli successfully isolated mechanisms sensitive to second-order motion.

To illustrate the findings of the individual patients and controls, Figure 5 depicts the distribution of log thresholds for direction discrimination (ordinate) plotted against the log thresholds for orientation identification (abscissa). The open symbols present 


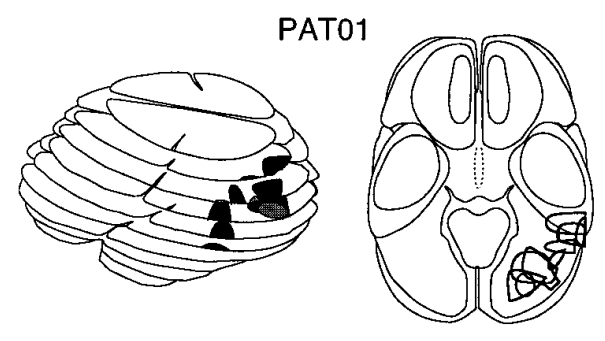

PAT02
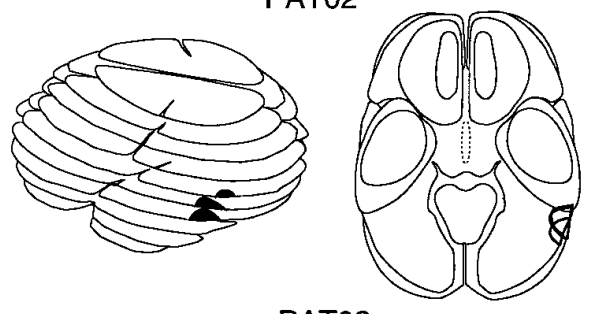

PAT03

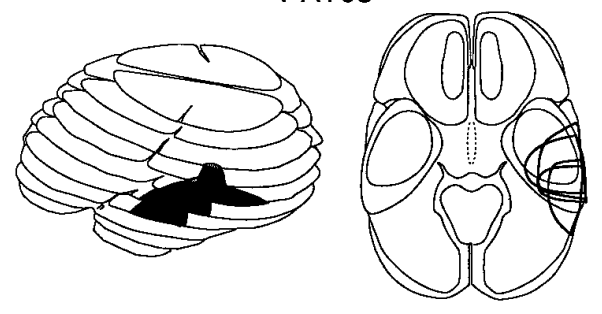

PAT04
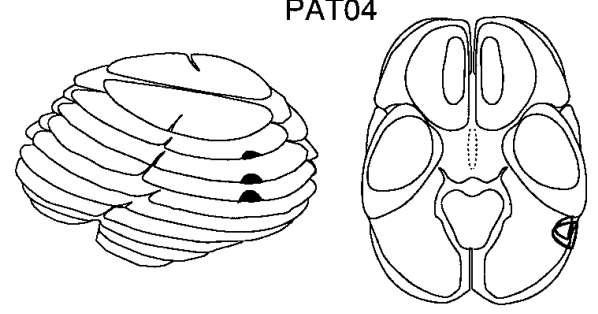

PAT05
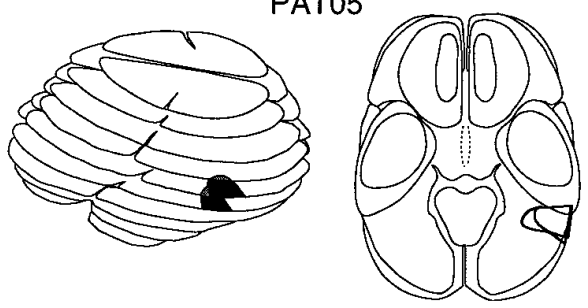

PAT06
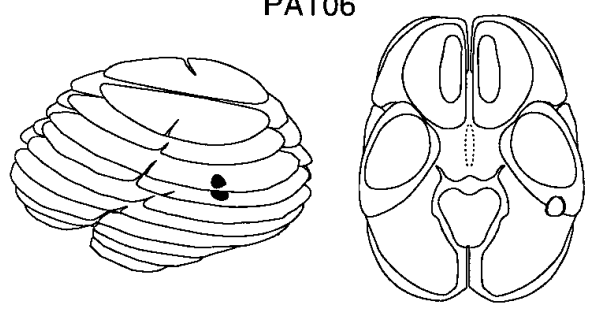
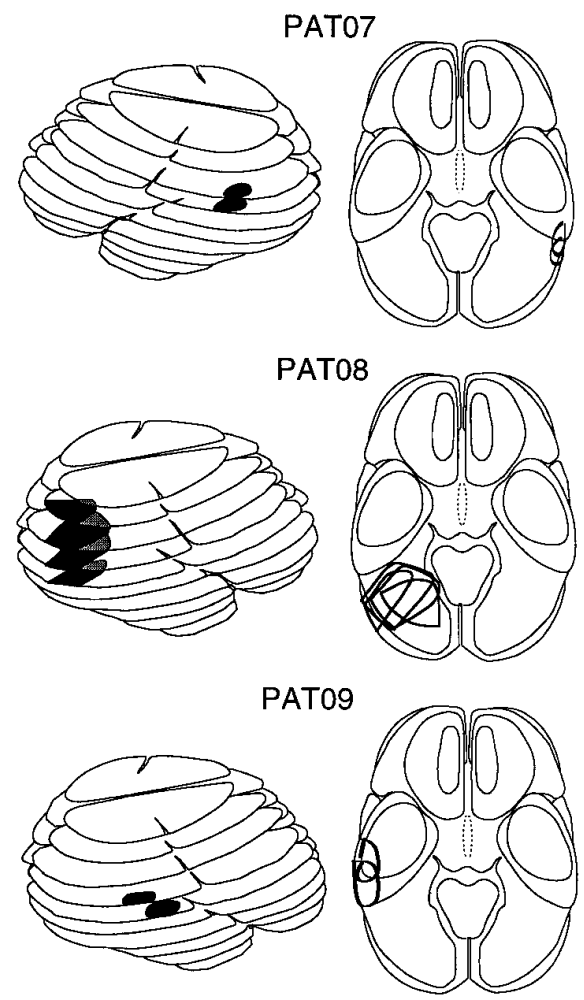

PAT10
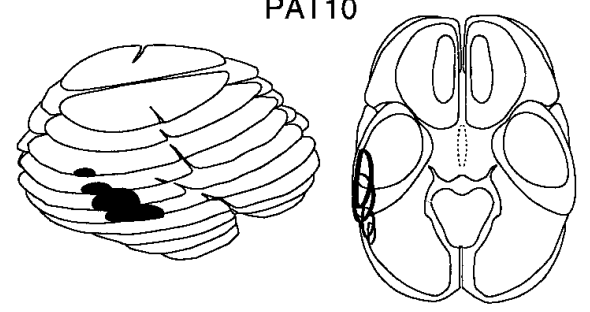

PAT11
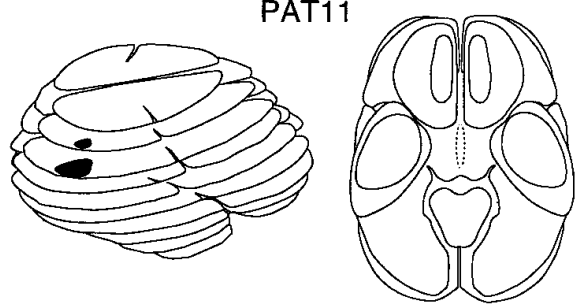

Figure 1. Schematic representation (lateral and axial views) of the computer tomograms of 11 patients with a lesion in the superior temporal cortex (a) and 10 patients with a lesion in the lateral inferoparietal cortex hemisphere (LIP group, $n=4$ ) or inferotemporal cortex (IT group, $n=6$ ). Dark regions denote the location of the lesion; gray areas depict the medial extent of the lesions. Figure continues.

the results for individual patients, and the type of symbols signifies the lesion location in that patient. The control thresholds are given by the filled dots. The $45 \mathrm{deg}$ line depicts the value by which thresholds for both types of discrimination are the same. Although several patients ( 7 of 21 ) show some threshold elevations for first-order motion $(a)$, almost all values fall along the unity line. For the patients showing elevated thresholds, this trend indicates that both orientation and direction discrimination are affected equally. The trend in the second-order motion thresholds is different from those of first-order motion $(b)$. Here, all thresh- 
PAT12
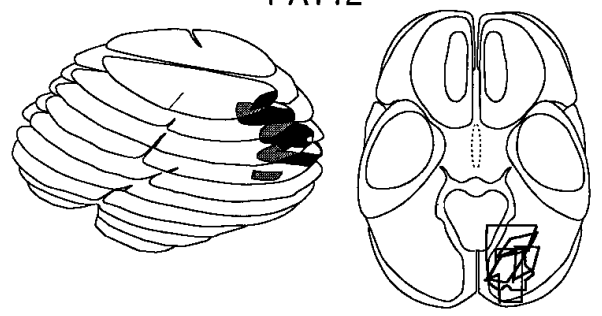

PAT13
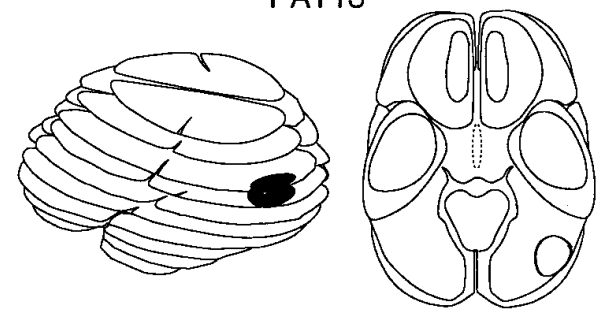

PAT14
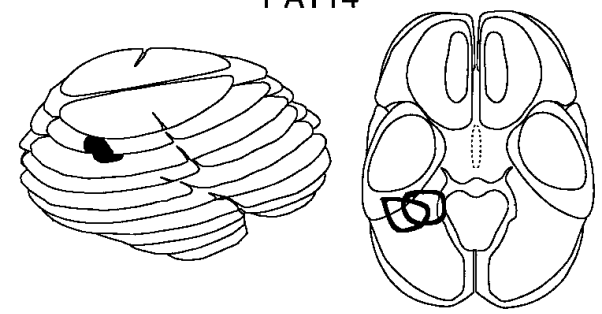

PAT15
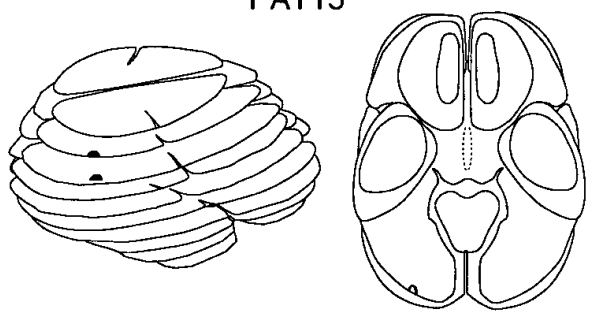

PAT16
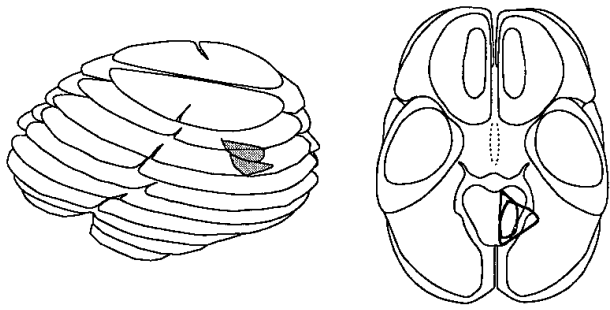

PAT17
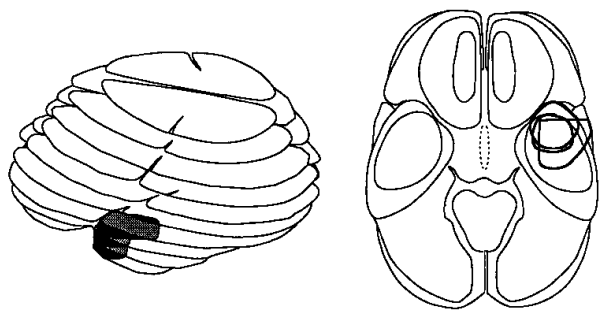

PAT18
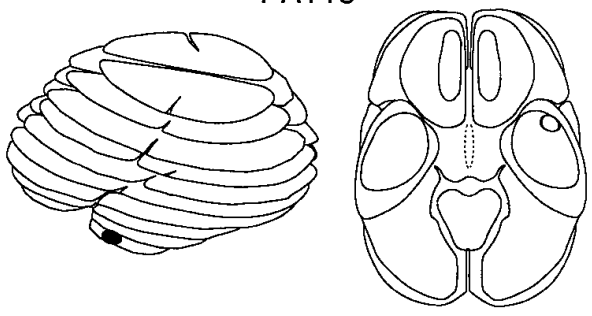

PAT19
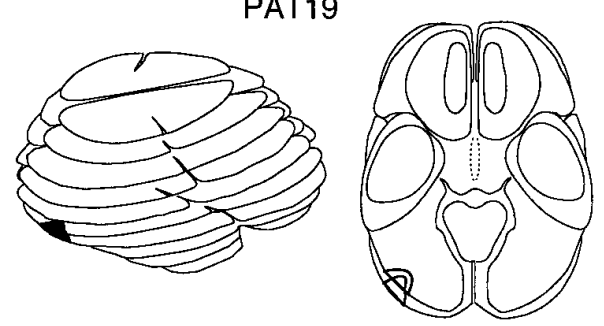

PAT20
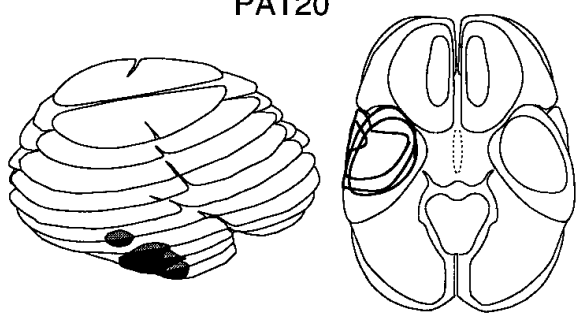

PAT21
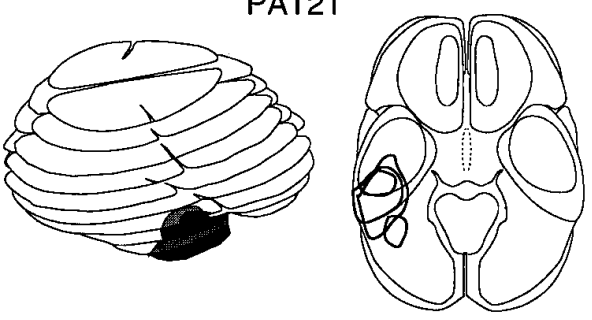

Figure 1 continued.

olds lie above the unity line; thus, the correct discrimination of direction required more contrast than that for orientation. There was a significant effect of lesion location on the log of the ratio of direction and orientation discrimination thresholds, $\left(\log \left(D_{\text {thres }} /\right.\right.$ $\left.O_{\text {thres }}\right)$, for second-order $\left(F_{2,60}=4.18 ; p=0.02\right)$, but not for first-order, motion $\left(F_{2,60}=2.19, p>0.1\right)$. Thresholds for direction discrimination were a factor of 2.3 higher in the ST patients and 2.44 times higher in the LIP patients as compared with orientation thresholds. IT patients showed direction thresholds that were 1.6 times higher than those for orientation and, as such, were more similar to the effect shown by the controls. Also, the speed of the patterns affected the direction/orientation threshold relationship, but again, this was only significant for the second- order motion stimuli $\left(F_{2,60}=3.36 ; p=0.04\right)$. At the lowest speed used $(1.5 \mathrm{deg} / \mathrm{sec})$, the patients required 2.6 times more modulation depth to discern correctly the direction of the drifting secondorder patterns, whereas at the two higher speeds this factor was reduced for both speeds to a factor of 1.9. This finding is in line with Smith and Ledgeway (1997).

Note that 8 of 14 controls and 7 of 21 patients show mean direction thresholds that are slightly lower than orientation thresholds for first-order motion $(a)$. Although these differences are small and not significant, they reflect the way in which we independently scored the response for orientation and direction. The instruction made clear to the participants that there were two alternatives for each type of response and that they would be 


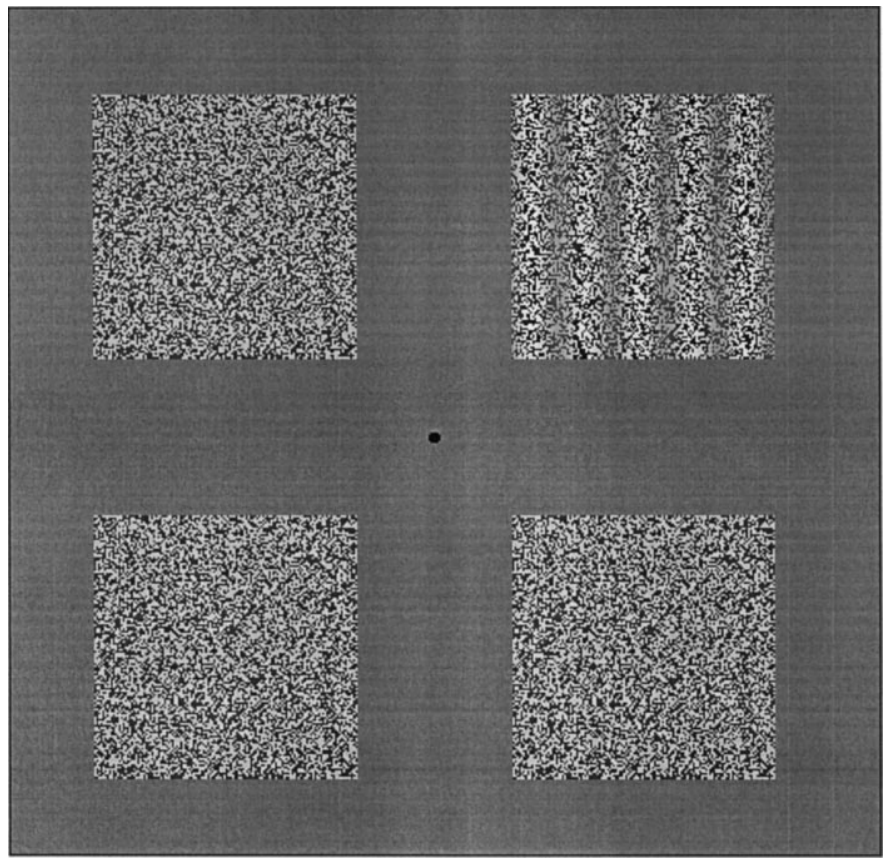

Figure 2. An illustration of the experimental paradigm and the stimuli used to determine orientation and direction identification thresholds and, in a modified form, speed discrimination thresholds (see text).

scored separately. Thus, for a given contrast level, the participant might respond incorrectly with respect to the orientation of the grating but correctly for its class of direction (left-up or rightdown). Similar findings have been reported for the detection and identification of stationary grating patterns when concurrent judgments were used (Thomas, 1983, 1985).

\section{Speed discrimination of first- and second-order stimuli}

The results of the speed discrimination experiments are shown in Figure $6 a$. The findings for the three patient groups and the controls are shown by open and filled symbols for first-order and second-order stimuli, respectively. Weber fractions $(\Delta \mathrm{V} / \mathrm{V})$ are shown as a function of the visual field condition with respect to the lesioned hemisphere. The patients exhibit large elevations in the speed discrimination thresholds. Patients with ST or LIP damage, in particular, show marked threshold elevations for first-order motion stimuli. Second-order speed discrimination thresholds also are elevated but to a lesser extent. There is no consistent effect of visual field, with the exception that ST patients show slightly lower thresholds for first-order stimuli in the ipsilesional visual field. The IT patients show effects that are less pronounced. Note that discrimination thresholds in the controls are somewhat lower for second-order stimuli than for first-order. This effect most likely is related to our choice of stimulus contrast for this experiment (see below).

The Weber fractions for the control subjects are substantially higher than those reported by others for healthy observers. Velocity Weber fractions for grating stimuli can be as low as 0.05 in foveal vision (McKee et al., 1986; Smith, 1987), although they increase with increasing eccentricity (Johnston and Wright, 1985). We attribute the higher Weber fractions $(\sim 0.3)$ found here to a combination of several factors. The main contributors are probably the eccentric viewing and the lack of practice of the observers. Other factors include the relatively low contrast and the uncertainty of position. The random jitter introduced on a trial- to-trial basis to the reference velocity also adds to the stimulus uncertainty, a factor which has been shown to increase detection and discrimination thresholds (Pelli, 1985; Graham, 1989).

An ANOVA was conducted to assess the effect of experimental group (patients vs controls), as well as the effects of motion type (first-, second-order) and visual field. The main effect of experimental group was highly significant $\left(F_{1,33}=32.17, p=0.0001\right)$. The patients exhibited, on average, thresholds that were 2.4 times higher than those of the controls. The main effect of visual field was not significant $\left(F_{3,99}=0.37, p=0.95, \mathrm{~ns}\right)$, whereas the effect of type of motion was highly significant $\left(F_{1,33}=30.9, p=0.0001\right)$. Thresholds for first-order motion were, on average, 1.44 times higher than for second-order motion. It should be noted that this is true of the controls as well as the patients. Discrimination performance is dependent on contrast, and the intention was to equate Weber fractions (for controls) across the two types of motion by manipulating contrast (see Materials and Methods). Clearly, they were equated imperfectly, and this makes the statistical significance of the main effect hard to interpret. More meaningful is the interaction between type of motion and experimental group, which was highly significant $\left(F_{1,33}=9.65, p=0.004\right)$, substantiating the impression given in Figure $6 a$ that thresholds for first-order motion were more elevated than for second-order motion in the patients, even allowing for the imperfect matching of contrast.

\section{Comparison of first-order and second-order speed discrimination thresholds}

Although the above statistical analysis indicates that speed discrimination with first-order motion was more impaired than that with second-order motion, it is not clear that a simple analysis of Weber fractions is the most appropriate approach. It could be argued that, because performance for the two image types is different in controls, this factor is better examined in terms of the ratio of the Weber fraction for a given patient group to that obtained by the control group using the same type of stimulus. For example, if second-order performance is $50 \%$ worse in a given patient group than in controls, it must be shown that performance is significantly $>50 \%$ worse than controls for first-order motion before it can be asserted that the deficit is greater for first-order. Figure $6 b$, replots the results from Figure $6 a$ as an attenuation relative to the control group. The magnitude of the impairment in discrimination performance varies across the patient groups, but performance is consistently worse for first-order than for secondorder. An ANOVA based on the magnitude of the impairment in $\mathrm{dB}$, rather than the raw $\Delta \mathrm{V} / \mathrm{V}$ values, showed a significant effect of type of motion $\left(F_{1,18}=4.5, p=0.048\right)$ but no interaction between type of motion and lesion location $\left(F_{2,18}=0.1\right.$, ns $)$.

Viewed in this way, the difference between performance on the speed discrimination of first-order and second-order motion, though consistent, is only moderate in magnitude. To explore further the extent to which first and second-order motion perception are dissociated in these patients, we performed a regression analysis between the individual means for first- and second-order speed discrimination thresholds. The results of this analysis are shown in Figure 7. The scatterplot shows the mean $z$ scores for each patient individually, and the number inside each symbol depicts the number assigned to each patient in Table 1 and Figure 1. The filled circles present the results for the control subjects. The $z$ scores are based on the mean and SD of the control group, calculated for each type of motion separately, in which the point of origin corresponds to the mean threshold for first (abscissa) 
a)

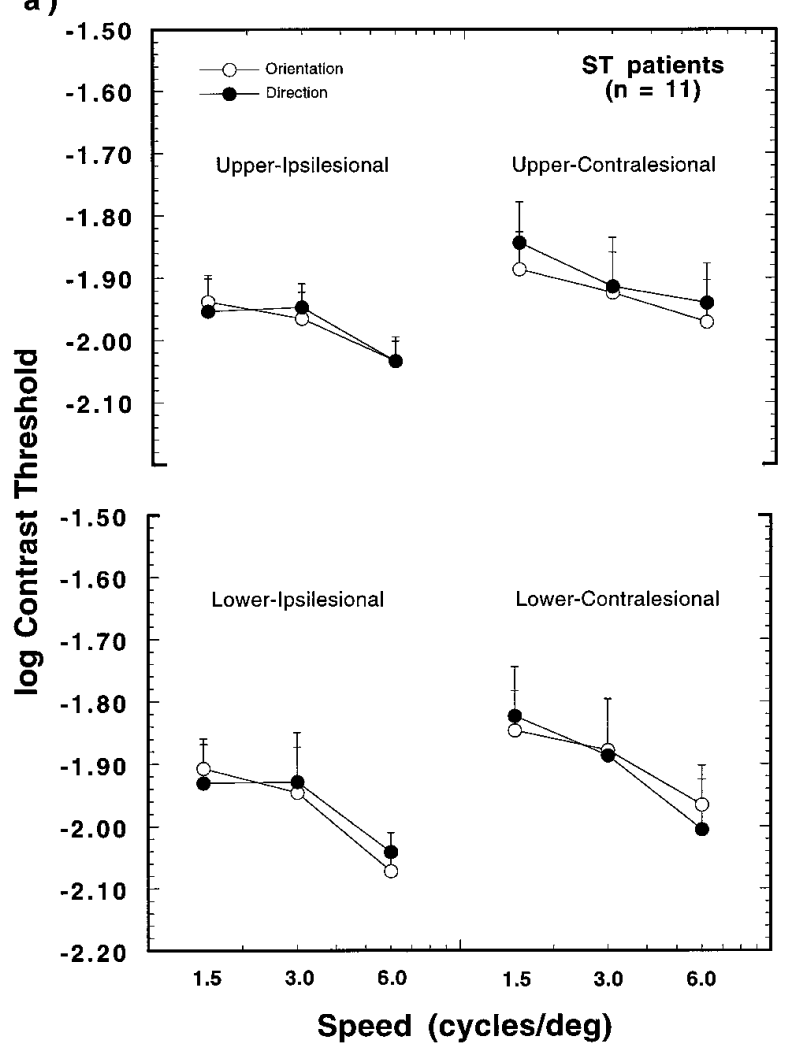

c)

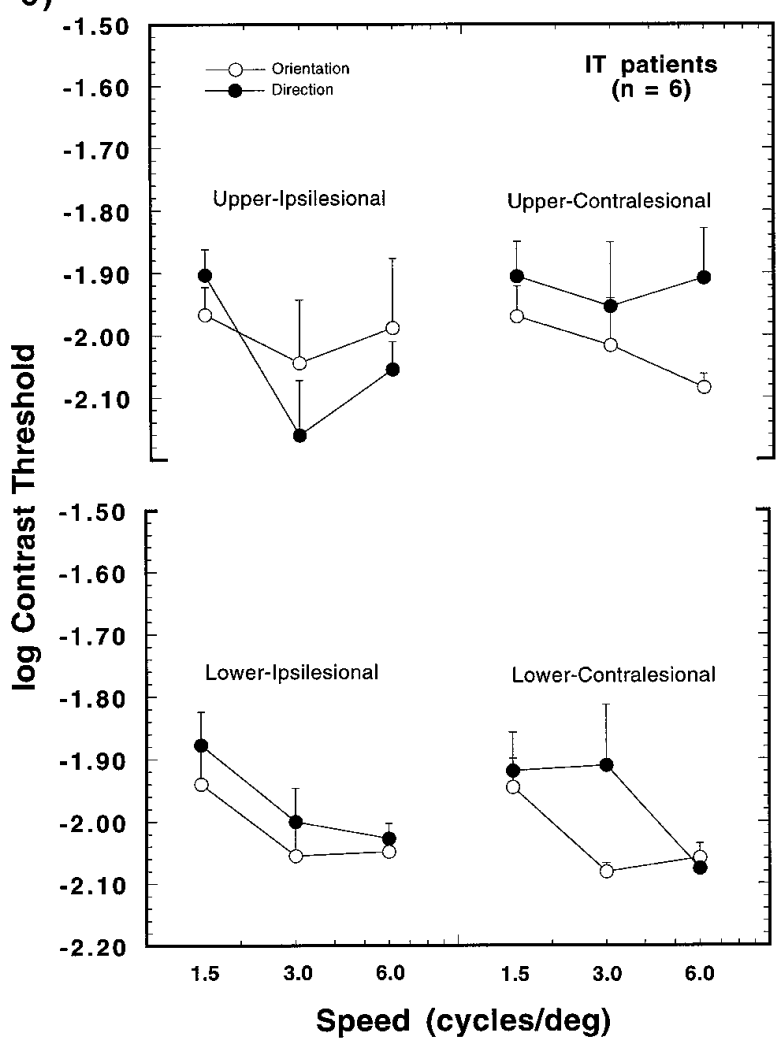

b)
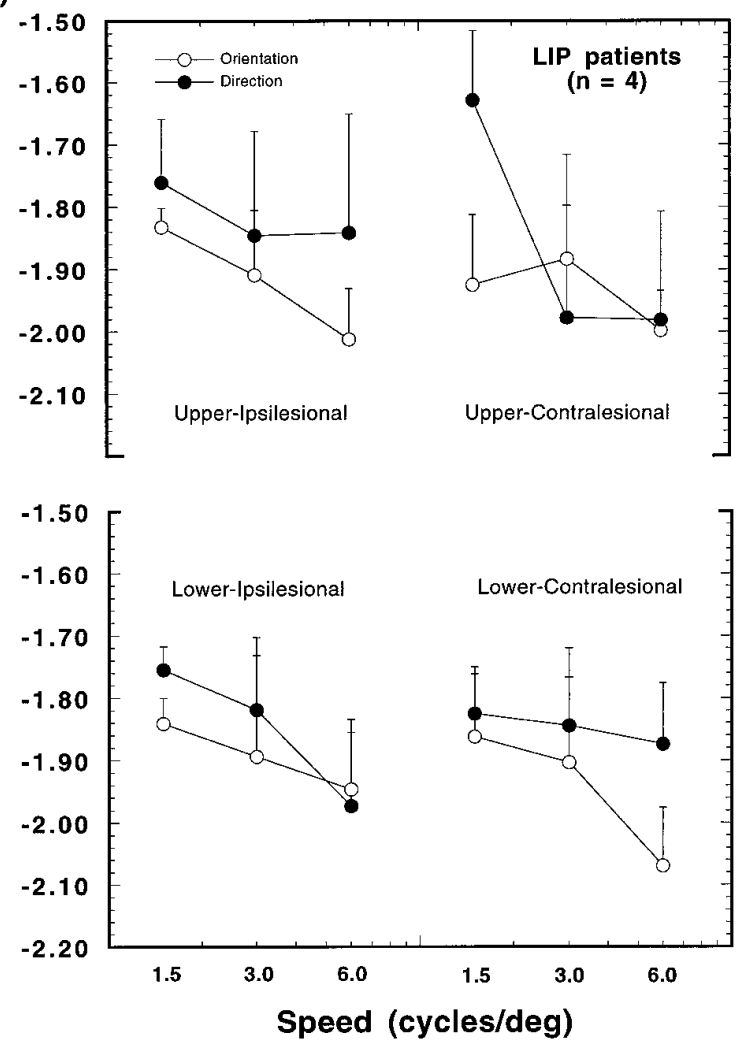

d)

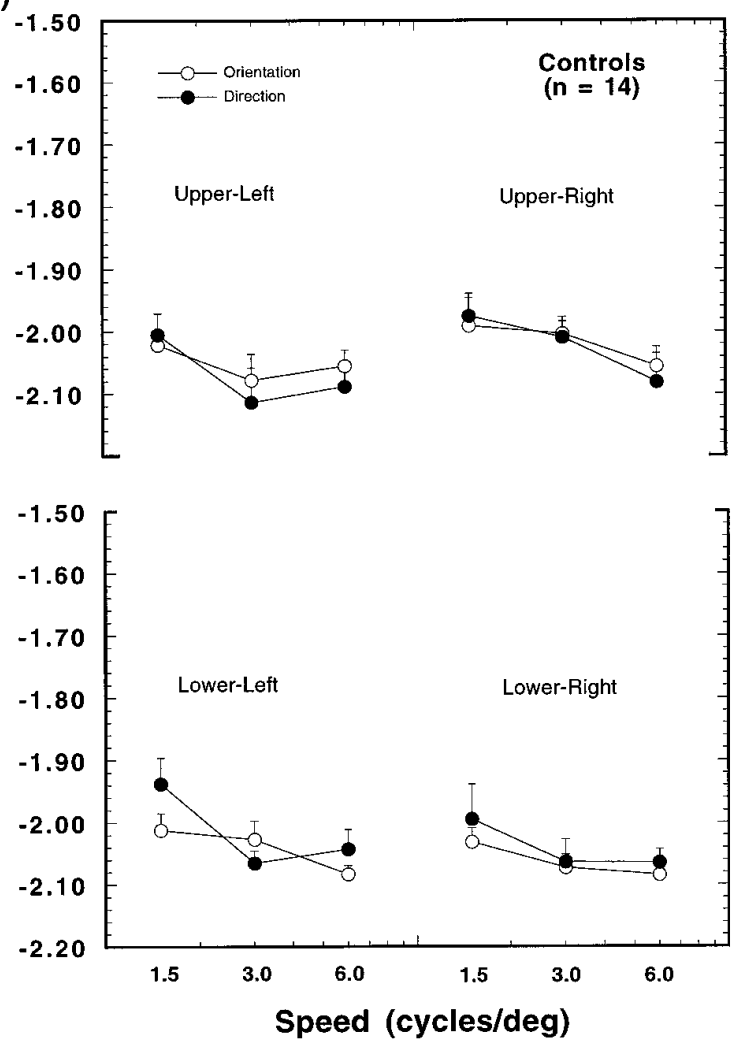

Figure 3. Mean log contrast thresholds for first-order stimuli presented in one of four visual quadrants (upper ipsilesional, upper contralesional, lower ipsilesional, lower contralesional). The results are plotted as a function of reference speed. Open and filled symbols give the mean thresholds for orientation and direction discrimination, respectively. $a$, Findings for 11 patients with damage in the region of the superior-temporal/occipital border (ST). $b$, The results for four patients with lateral inferoparietal lesions $(L I P) . c$, The findings for six patients with damage in inferior temporal cortex $(I T)$. $d$, The results for the 14 control subjects. Error bars show +1 SEM thresholds, averaged over subjects. 
a)

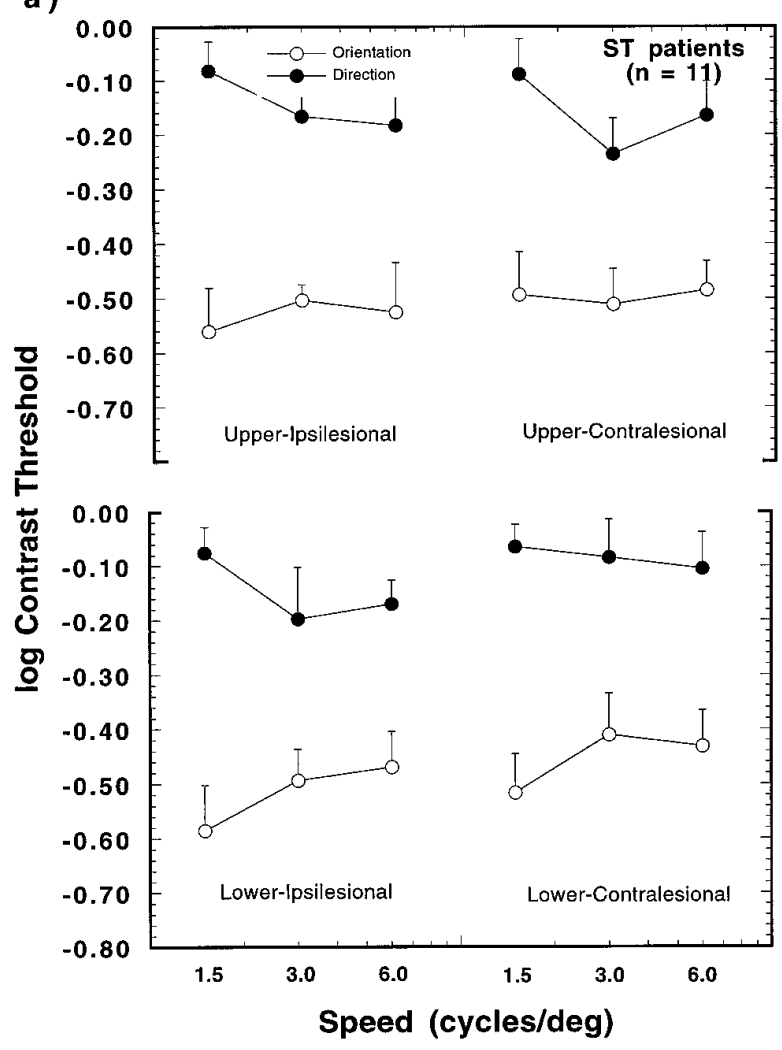

c)

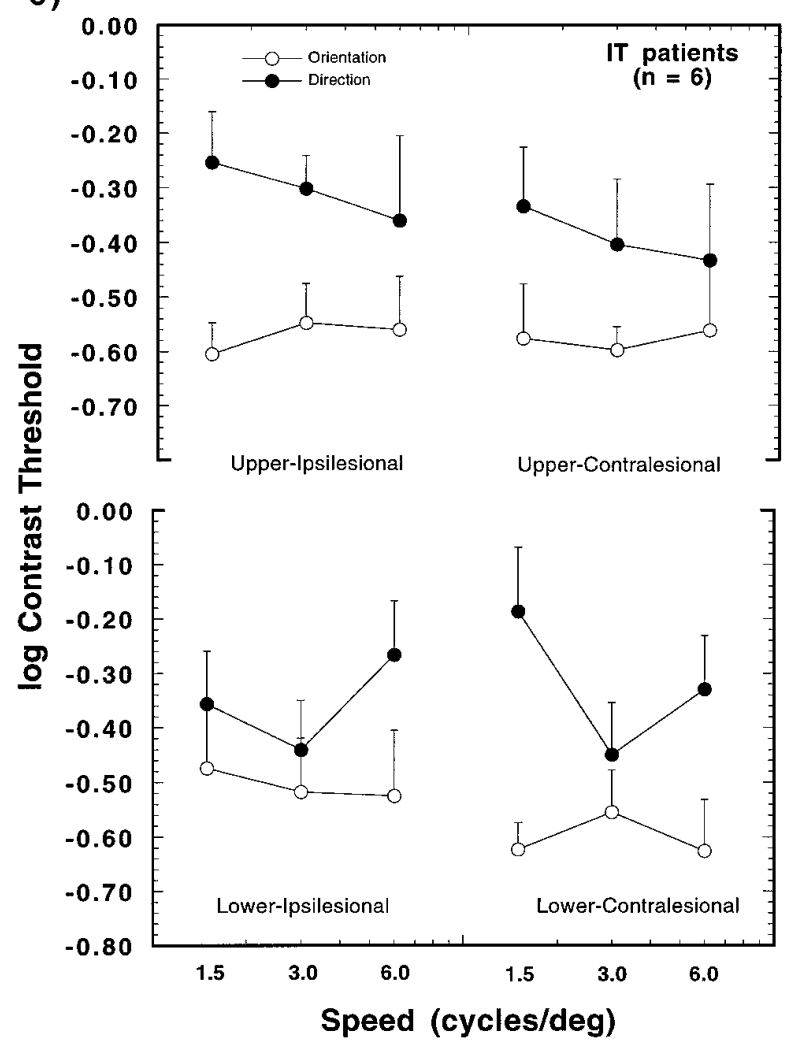

b)
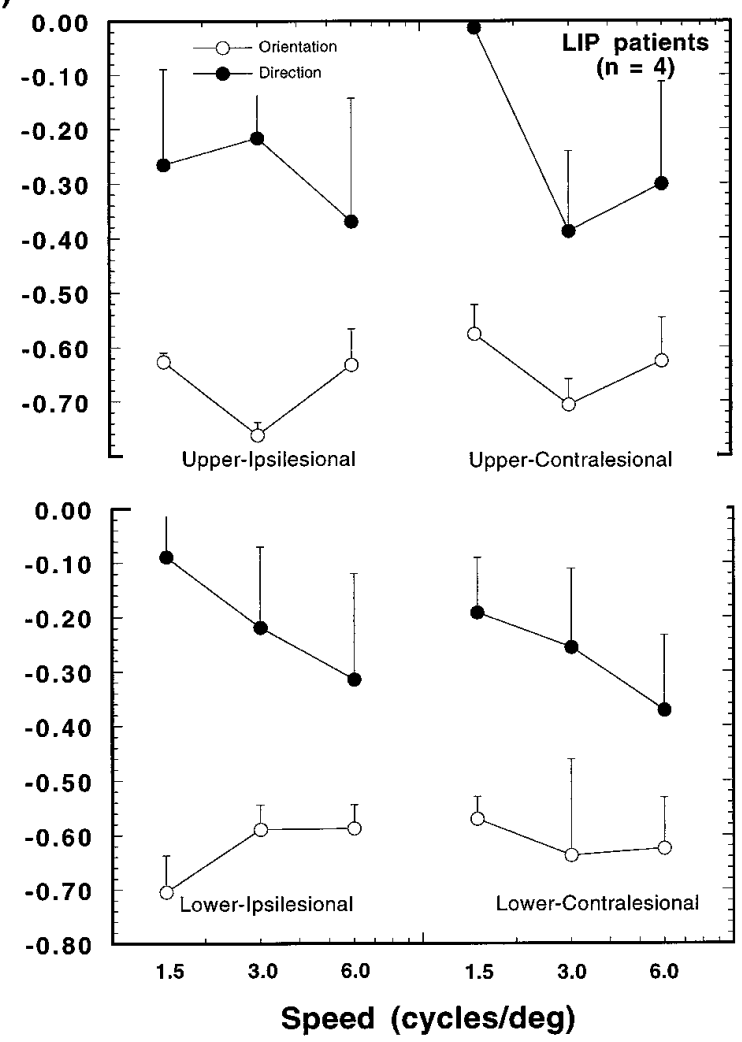

d)

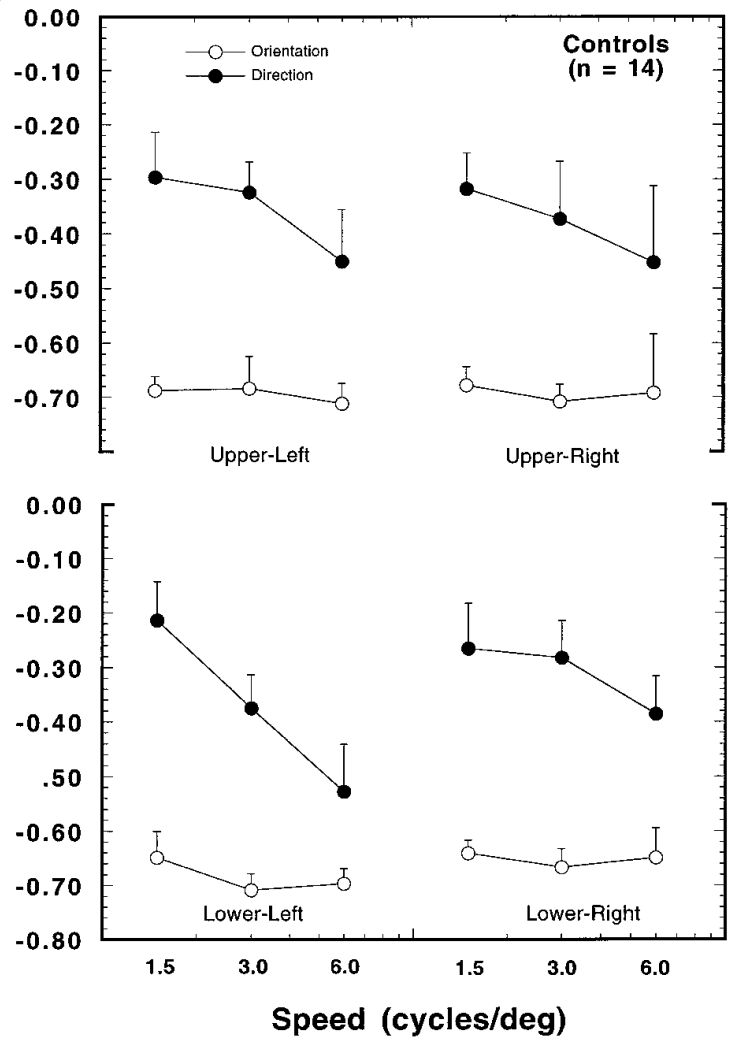

Figure 4. Mean log contrast thresholds for second-order stimuli; otherwise as in Figure 3. 
a)

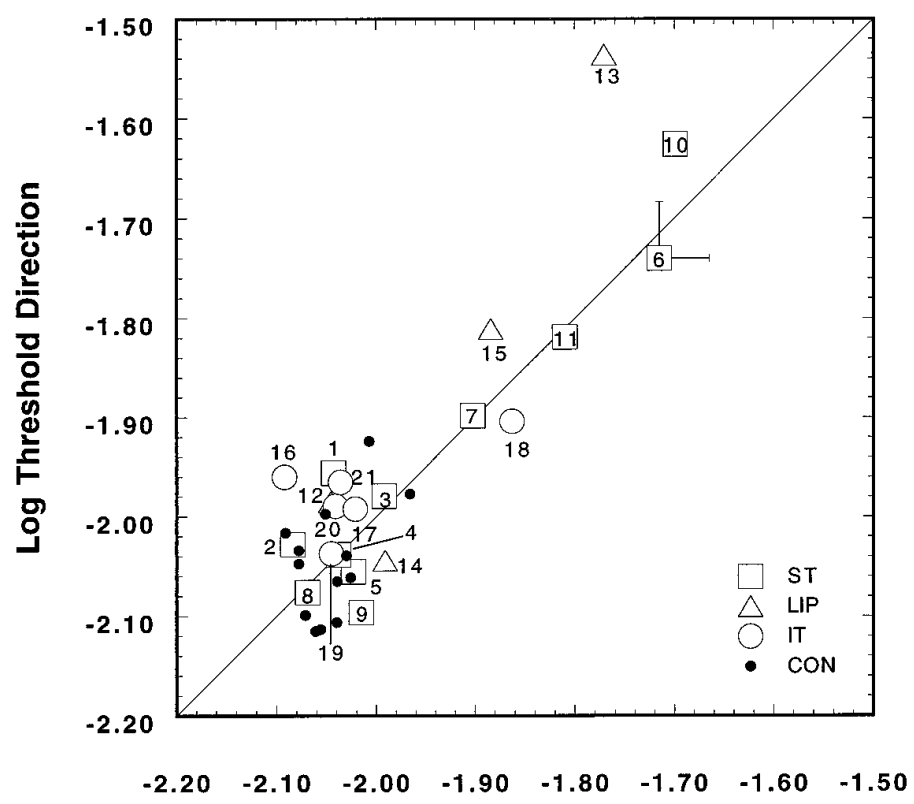

b)

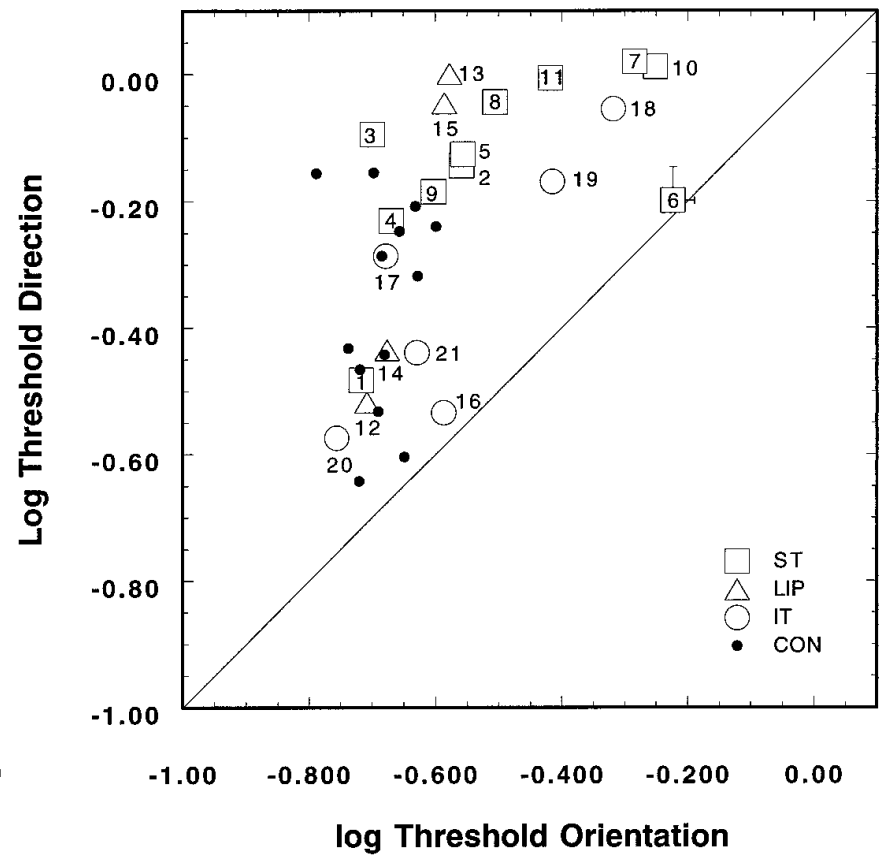

Figure 5. Mean log contrast thresholds for first-order $(a)$ and second-order $(b)$ stimuli, replotted from Figures 3 and 4 to show the individual variation among the patients and controls. Log thresholds for orientation identification are plotted against the log of the direction discrimination threshold (averaged over speeds and visual quadrants for each participant). The error bars (shown together with the value for PAT06) present the average SEM values (averaged over patients) for both types of judgment.

and second-order (ordinate) motion. The regression of the $z$ scores for second-order speed discrimination onto the $z$ scores for first-order speed discrimination is highly significant $(R=0.812$, $p=0.0001)$. Thus, $\sim 66 \%$ of the variance in the second-order speed thresholds (i.e., $R^{2}$ ) can be accounted for by the variance in first-order thresholds.

\section{Effect of lesion location on discrimination thresholds}

The results shown in Figure 6 suggest that patients with damage in the superior temporal and/or inferior parietal cortex are more impaired on speed discrimination tasks than are patients with inferotemporal damage. We performed a further ANOVA to test this possibility. We entered patient group (ST, IT, LIP) into the analysis for speed discrimination to assess its main effect on the variance in the thresholds, as well as its possible interactions with the other stimulus-related variables. The main effect of lesion location was moderately significant $\left(F_{2,18}=3.58, p=0.049\right)$. Speed discrimination thresholds were elevated more greatly in patients with ST and LIP damage than in those with IT damage.

\section{Cortical maps}

The results presented in Figure $6 a, b$ are averaged across patients who were assigned to one of three groups on the basis of fairly broad criteria. In reality, of course, the lesions vary substantially within each of the three groups in terms of both location and extent. To analyze the results in a way that takes fuller account of the location and extent of each lesion, we have collapsed the results of all 21 patients into a single cortical map that shows the performance deficit associated with a lesion in each location in the cortex. Separate maps were computed for first- and secondorder motion. The results of this analysis are shown in Figure 8 . For each patient, speed discrimination performance, averaged across the four visual field locations, was expressed as a single $z$ score for each of the 21 patients. Using the $10 \times 12$ matrix of cortical locations shown in the left half of Figure 8 , we then calculated for each cell in the matrix (each cortical location) the average $z$ score of all patients whose lesion included that location. Then the results for each cell were weighted by the square root of the number of patients whose $z$ score contributed to that cell, to emphasize trends that are consistent across patients. The gray levels shown in the two maps represent the normalized values for each type of motion. The normalization was performed solely on the mean and SD of the patient group for each type of motion separately, without reference to the control data. This method differs slightly from that originally used by us (Greenlee et al., 1995), in that the value is normalized within the patient group and each value is weighted by the number of observations. We believe that this procedure more clearly illustrates consistent differences among patients. Inspection of both maps reveals a marked similarity between the effect of the lesion location on performance for both types of motion stimuli. The maximum value of the secondorder map is somewhat more anterior than that found for the first-order stimuli. There is also some indication of a second peak having a more dorsal and posterior location.

\section{DISCUSSION}

The results of the present investigation indicate that damage in the superior temporal/lateral occipital border region and/or the lateral inferior parietal cortex leads to an impairment in motion perception. By analyzing discrimination performance for orientation, direction, and relative speed of first- and second-order stimulus motion, we have quantified the extent to which motion perception is impaired in this patient sample. 

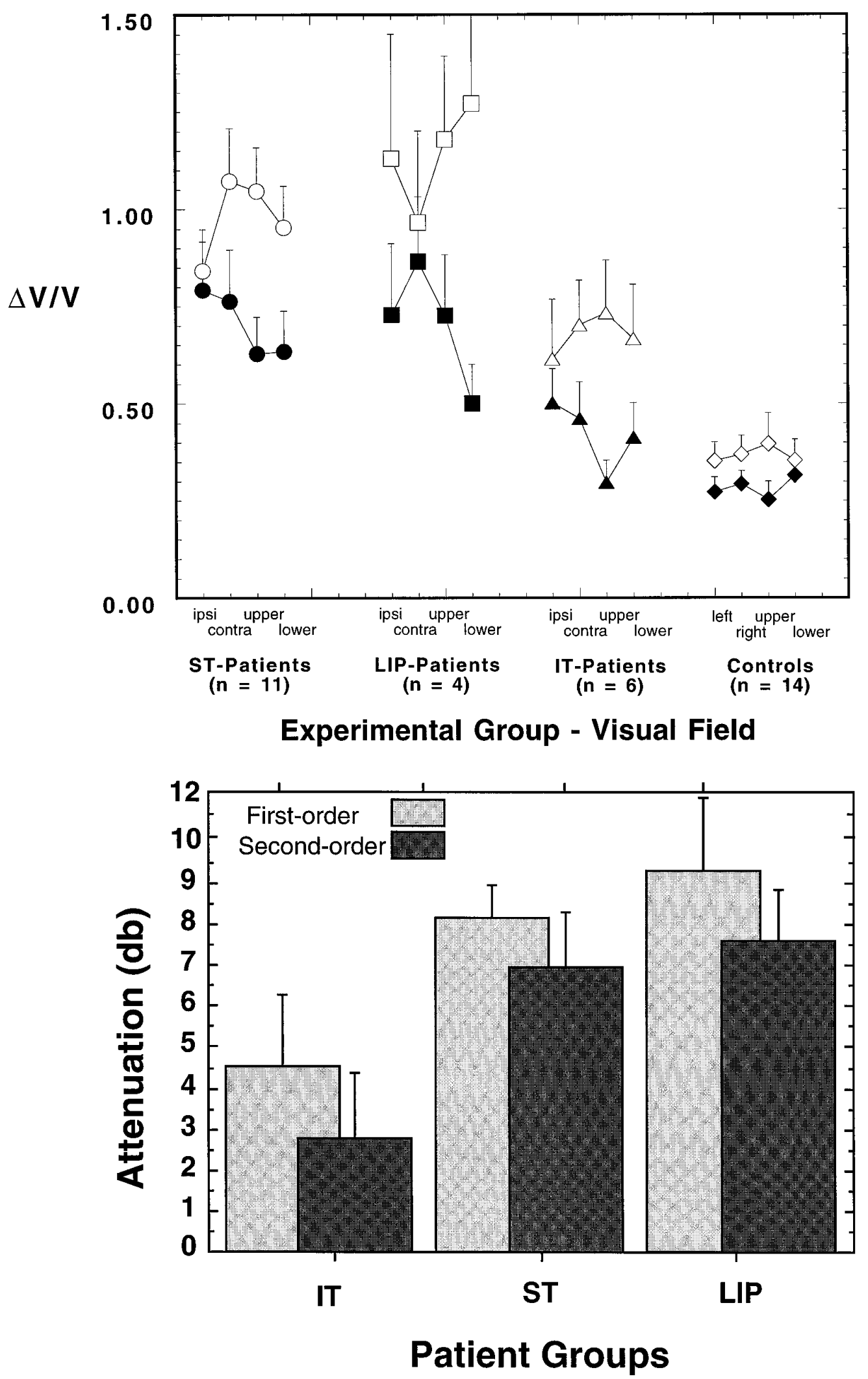

Figure 6. a, Mean speed discrimination performance $(\Delta \mathrm{V} / \mathrm{V})$ for first-order (open symbols) and second-order ( filled symbols) motion as a function of the visual field of presentation (ipsilesional, contralesional, upper, and lower). Error bars show 1 SEM (averaged over subjects and stimulus directions). The results are shown separately for the $S T, L I P$, and $I T$ groups. The values for the controls are shown to the right. $b$, The same data expressed in terms of the magnitude of the performance deficit in patients relative to controls. The mean speed discrimination performance for each patient group is shown, separately for first- and second-order, as an attenuation in $\mathrm{dB}$ relative to control performance. 


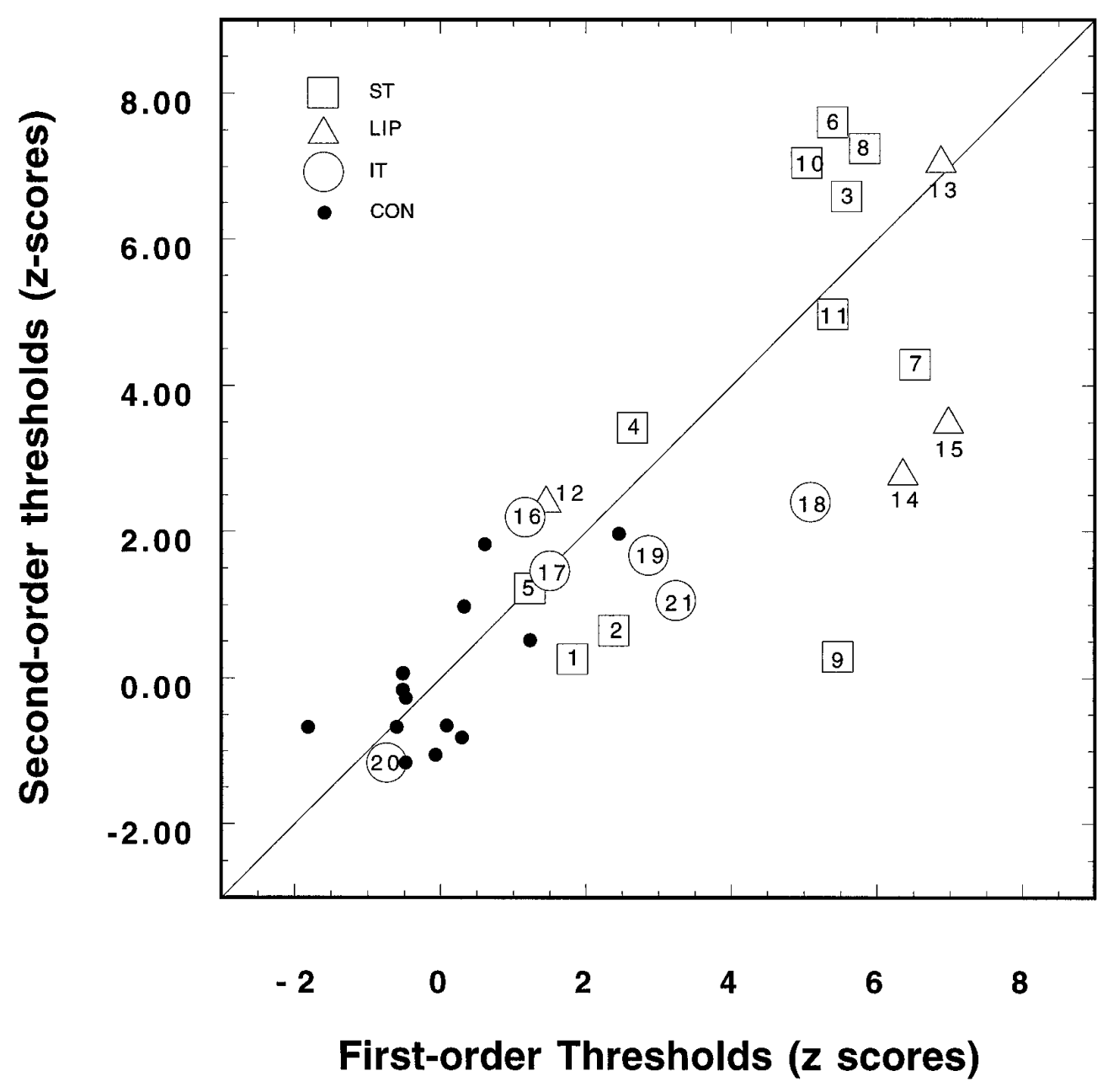

Figure 7. Scatterplot showing the normalized speed discrimination thresholds ( $z$ scores) measured for first-order stimuli (abscissa) and the corresponding thresholds determined for second-order stimuli (ordinate). Each patient's score is depicted by an open symbol containing the number assigned to the patient (see Table 1). The solid line shows a slope of 1.0. The regression $(b=0.81 \pm 0.1)$ of second order on first-order $z$ scores was $<1.0$.

\section{Orientation identification thresholds for first- and second-order motion}

The ability of the patients to identify the orientation of moving patterns remained, for the most part, intact. Four patients in the ST group and two patients in the LIP group did exhibit higher orientation thresholds than the controls for first-order stimuli (Fig. 4). Orientation discrimination for second-order stimuli was normal in patients with LIP lesions. Two patients with IT lesions (PAT18, PAT19) and three patients with ST lesions (PAT06, PAT07, PAT10) had elevated second-order orientation thresholds. Although the group mean difference is small (31\% increase for IT patients, $52 \%$ increase for ST patients), it suggests that damage in the temporal cortex can lead to a moderate impairment in the ability to extract orientation information for second-order motion cues. Interestingly, Sary and collaborators (1993, 1995) recently have reported evidence suggesting that the inferior temporal cortex in monkeys can extract information about the shape or orientation for objects defined by luminance, texture, or motion differences. It should be noted that any uncorrected refractive errors would affect the visibility of the high-pass-filtered noise carriers and, as such, would affect second-order thresholds more than first-order. All participants reported that they easily could detect the presence of the four static noise fields. Formal measurement of the patients'/controls' contrast sensitivity to the noise patches alone was not, however, conducted. The moderate elevations in orientation thresholds could, in part, be related to uncorrected refractive errors.

\section{Direction discrimination of first- and second-order motion}

The experimental paradigm used in the present investigation allowed us, in addition to orientation discrimination, to determine simultaneously the thresholds for detecting the direction of motion of the patterns. The results, shown together with the orientation thresholds in Figures 3 and 4, indicate that patients in the ST and LIP groups require greater levels of contrast modulation than the control group to discriminate the direction of moving second-order patterns reliably. Patients in the ST group exhibited thresholds that were $66 \%$ higher $(0.22 \mathrm{log}$ unit) than for the controls. LIP patients showed a smaller effect $(27.6 \% ; 0.11 \mathrm{log}$ unit). Although these effects are moderate in size and the variability among the patients is considerable, the ANOVA indicated that these effects are highly significant. This result suggests that direction discrimination was consistently impaired in the patients with ST and LIP lesions.

\section{Relationship between orientation and direction discrimination thresholds}

It may be fair to ask to what extent the effects shown for direction thresholds are truly motion-specific. Because the ST patients exhibited elevations in both orientation and direction, it could be argued that the effects of the one are transmitted to the other. Although we have scored responses on each dimension separately (see above), we cannot easily rule out that both types of threshold could have been affected by the visibility of the noise carriers. To 

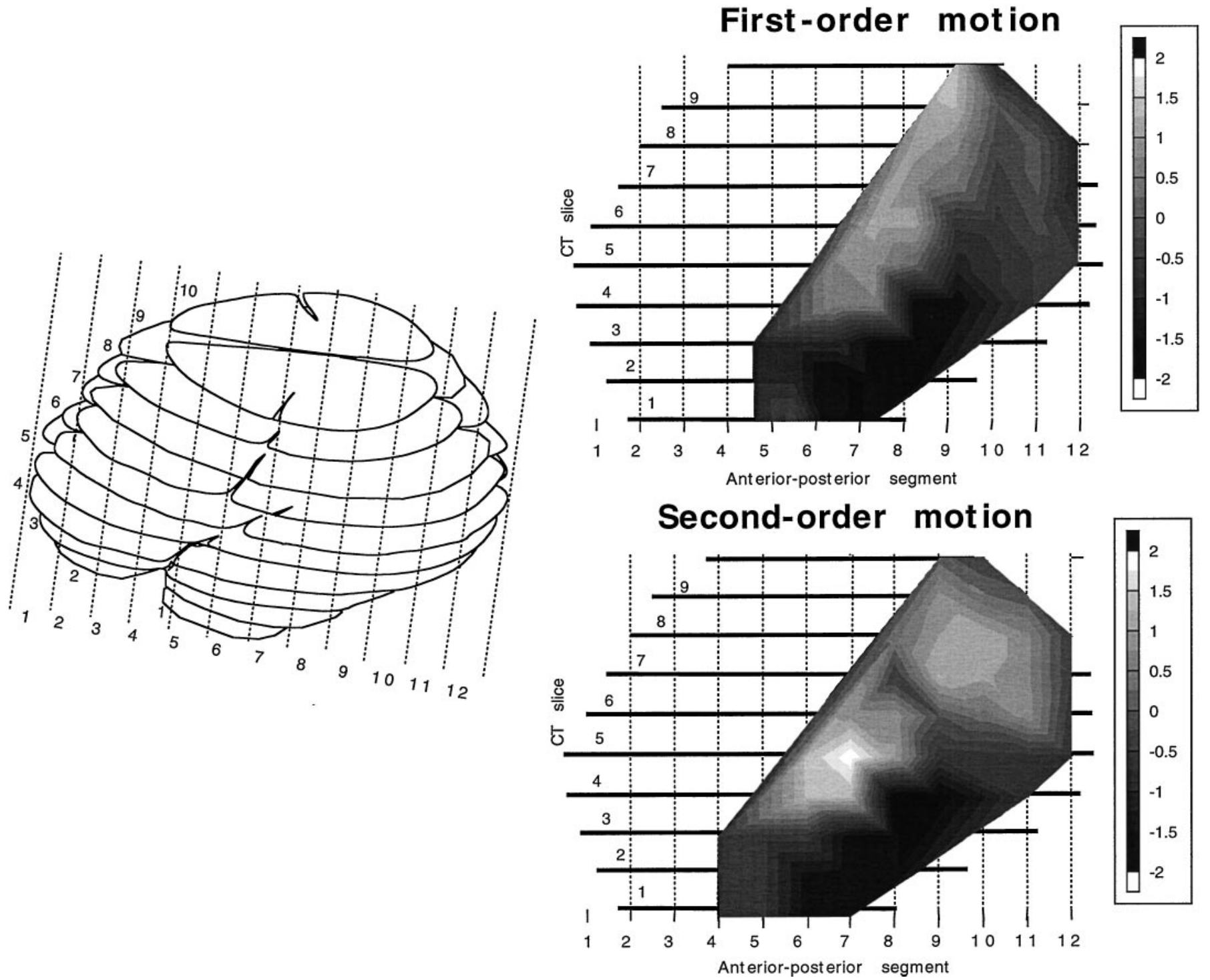

Figure 8. Computed tomographic map of the $z$-score-weighted location of the cortical lesions averaged over all 21 patients for first-order and second-order stimuli. The left panel shows a lateral view of the 10 computer tomographic slices, and the two right panels show the topographic distribution of the averaged $z$ scores. Light areas signify the lesion locations that were associated with the most pronounced impairments in speed discrimination.

examine this point in more detail, we analyzed the relationship between the orientation and direction thresholds and determined how this relationship was affected by a lesion in the posterior cortex (Fig. 4). We found a significant effect of lesion location on the $\log$ of the ratios for direction and orientation discrimination thresholds for second-order, but not for first-order, motion. Thus, direction impairment cannot be attributed entirely to factors that are not motion-specific, such as uncorrected refractive errors. Clearly several ST and LIP patients have greater difficulty discriminating the direction of the moving patterns than discriminating their orientation (Fig. 4).

\section{Speed discrimination of first- and second-order motion}

As can be seen in Figure 6, by far the most pronounced deficits we observed were in suprathreshold speed discrimination performance. Despite our use of a relatively high suprathreshold contrast (corresponding to 5-10 times detection threshold), patients in the ST and LIP groups exhibited speed discrimination thresh- olds that often rose above a Weber fraction of 1.0 (i.e., factor of 2.0 increase). Under the same conditions, age-matched controls exhibit Weber fractions that lie below 0.4. The perception of speed was more impaired for first-order motion than it was for second-order motion. Nonetheless, we found a significant correlation between the patients' thresholds for the two types of motion (Fig. 7). Thus, the dissociation suggested in Figures 6 and 7 is only partial, and the results suggest that, to a large extent, a common area or set of areas mediates our ability to discriminate the speed of the two types of motion.

\section{Effect of lesion location}

As evident in the findings shown in Figure 6, the location of the cortical lesion determined to some extent the effect it had on speed discrimination. To analyze further the role of the lesion location, we computed the maps shown in Figure 8. Following a method similar to that described in an earlier study (Greenlee et al., 1995), we mapped out the location of each patient's lesion into the 12 anterior-posterior segments of 10 computed tomographic 
layers. The two maps (Fig. 8) indicate that a lesion in the posterior part of the superior temporal gyrus and lateral inferior parietal cortex leads to a significant impairment in speed discrimination. A lesion in the inferotemporal gyrus had, in contrast, less effect on speed discrimination. The first-order map is similar to that reported by us earlier (Greenlee et al., 1995), with the difference that the maximum effect extends more dorsally. The map for second-order stimuli is, for the most part, comparable with respect to the location of a maximum in the superior temporal gyrus. On the basis of these findings, we conclude that perception of the speed of first- and second-order motion is mediated, for the most part, by mechanisms located in common extrastriate areas. However, in the second-order map in Figure 8, there is some indication of a second focus in a more dorsal-posterior part of parietal cortex (corresponding to the posterior supramarginal gyrus).

\section{Relation to earlier lesion studies}

Zihl and collaborators have reported a patient with extensive bilateral lesions in the posterior cortex (Zihl et al., 1983, 1991). MRI scans made in 1989 indicated bilateral damage of the middle temporal gyrus and adjacent occipital cortex. There was also evidence for a right-sided cerebellar lesion. This patient suffers from "motion blindness," which is evident in her inability to respond adequately to moving stimuli. She has difficulty discriminating the direction of moving bars (Zihl et al., 1983) and can reliably detect the direction of random dots only if most of them are moving coherently (Baker et al., 1991). She also appears to underestimate the speed of targets moving at rates above 6 $\mathrm{deg} / \mathrm{sec}$ (Zihl et al., 1991). Positron emission tomography scans of her brain made while she viewed motion sequences indicated that the cortical activation was shifted dorsally to a more parietal location in area 7 (Shipp et al., 1994). Our findings are broadly consistent with those of Zihl and colleagues (1983, 1991), in the sense that relatively small, unilateral lesions cause less severe impairments of a similar type to those caused by a large, bilateral lesion in the same region.

Plant and collaborators (Plant and Nakayama, 1993; Plant et al., 1993) have studied three patients extensively and have reported on their ability to discriminate the direction and speed of firstorder motion stimuli. They also studied the ability of these patients to discriminate the direction of a second-order pattern made of the sum of two oppositely drifting sine gratings (a "beat" stimulus). After resection of temporal-occipital cortex (cases 1-3, their Fig. 7), the direction discrimination for second-order motion was more impaired than for first-order motion. Although we also found direction discrimination to be more impaired for secondorder motion, speed discrimination clearly was more affected for first-order motion, suggesting a partial dissociation between the processing of direction and speed.

Plant et al. (1993) also examined four parietal lobe patients who showed no effect on their detection/discrimination ratio index. In an earlier study, we (Greenlee et al., 1995) also found little or no deficit in speed perception in four parietal lobe patients. These earlier results are in contrast to the present finding that patients with damage in the inferoparietal cortex exhibited large impairments in their ability to discriminate the speed of first-order and, to a lesser extent, second-order motion (Fig. 5). The difference in results could be related to the paradigm used in the present study, which encorporated random locations of reference and test stimuli.

Lesions in the macaque medial superior temporal area (MST) lead to an impairment in smooth pursuit eye movements (Kom- atsu and Wurtz, 1988a,b; Newsome et al., 1988). The studies by Newsome et al. (1985) and Newsome and Paré (1988) have shown that lesions in V5/MT lead to an impairment in the ability of monkeys to detect and discriminate motion stimuli. Pasternak and Merigan (1994) also have reported that an experimentally induced lesion in V5/MT leads to an impairment in direction and speed discrimination in monkeys. The size of these effects was moderate, suggesting a role for other cortical regions in the discrimination of stimulus speed. In a separate investigation, Kimmich and coworkers (1995) measured smooth pursuit eye movements in patients with ST lesions. They found that the gain of smooth pursuit was reduced by $\sim 30 \%$.

Can second-order motion be processed in the absence of firstorder motion-detecting mechanisms? Our findings suggest that, although there seems to be much in common between mechanisms processing these two types of motion, there are also some differences. Individual patients (PAT09, PAT14, PAT15; Fig. 7) had thresholds for first-order motion discrimination that were much more elevated than those for second-order motion. This sort of result would speak against an hierarchical organization and in favor of parallel detection of first- and second-order motion. Other patients (PAT03, PAT06, PAT08, PAT10, PAT13) show large impairments in both first- and second-order speed discrimination. We have not yet found a patient with severely impaired second-order speed perception but intact first-order speed discrimination. All of the stimuli used in the present study contained carriers with static random dot noise. Motion also can be detected in dynamic noise, and lesions in the human TPO area (as well as the macaque MT/MST complex) could have large effects on this performance, as suggested by a recent report (Rudolph and Pasternak, 1996). An important extension to this investigation would be to determine whether patients with TPO lesions have more difficulty with tasks involving motion perception in dynamic noise fields.

\section{REFERENCES}

Adelson EH, Bergen JR (1985) Spatiotemporal energy models for the perception of motion. J Opt Soc Am A 2:284-299.

Albright TD (1984) Direction and orientation selectivity of neurons in visual area MT of the macaque. J Neurophysiol 52:1106-1130.

Baker CL, Hess RF, Zihl J (1991) Residual motion perception in a "motion-blind" patient, assessed with limited lifetime random dot stimuli. J Neurosci 11:454-461.

Chubb C, Sperling G (1988) Drift-balanced random stimuli: a general basis for studying non-Fourier motion perception. J Opt Soc Am A 5:1986-2006

Derrington AM, Badcock DR (1985) Separate detectors for simple and complex patterns? Vision Res 25:1869-1878.

Foster DH, Bischof WF (1991) Thresholds from psychometric functions: superiority of bootstrap to incremental and probit variance estimators. Psychol Bull 109:152-159.

Graham N (1989) Visual pattern analyzers. New York: Oxford UP.

Greenlee MW, Lang H-J, Mergner T, Seeger W (1995) Visual short-term memory of stimulus velocity in patients with unilateral posterior brain damage. J Neurosci 15:2287-2300.

Johnston A, Wright MJ (1985) Lower thresholds of motion for gratings as a function of eccentricity and contrast. Vision Res 25:179-185.

Kimmich H, Pinow C, Mergner T, Greenlee MW (1995) Smooth pursuit eye movements in patients with impaired visual motion perception. In: Multisensory control of posture (Mergner T, Hlavacka F, eds), pp 325-329. New York: Plenum.

Komatsu H, Wurtz RH (1988a) Relation of cortical areas MT and MST to pursuit eye movements. III. Interaction with full-field visual stimulation. J Neurophysiol 60:621-644.

Komatsu H, Wurtz RT (1988b) Relation of cortical areas MT and MST to pursuit eye movements. I. Localization and visual properties of neurons. J Neurophysiol 60:580-603. 
Maunsell JHR, Van Essen DC (1983) Functional properties of neurons in the middle temporal visual area of the macaque. I. Selectivity for stimulus direction, speed, and orientation. J Neurophysiol 49:1127-1147.

McKee SP, Silverman GH, Nakayama K (1986) Precise velocity discrimination despite random variations in temporal frequency and contrast. Vision Res 26:609-619.

Mikami A, Newsome WT, Wurtz RH (1986a) Motion selectivity in macaque visual cortex. I. Mechanisms of direction and speed selectivity in extrastriate area MT. J Neurophysiol 55:1308-1327.

Mikami A, Newsome WT, Wurtz RT (1986b) Motion selectivity in macaque visual cortex. II. Spatiotemporal range of directional interactions in MT and V1. J Neurophysiol 55:1328-1351.

Movshon JA, Adelson EH, Gizzi MS, Newsome WT (1986) The analysis of moving visual patterns. Exp Brain Res [Suppl] 11:117-152.

Nadjmi M, Piepgrass U, Vogelsang H (1991) Kranielle Computertomographie. Stuttgart: Thieme.

Newsome WT, Paré EB (1988) A selective impairment of motion perception following lesions of the middle temporal visual area (MT). J Neurosci 8:2201-2211.

Newsome WT, Wurtz DH, Dürsteler MR, Mikami A (1985) Deficits in visual motion processing following ibotenic acid lesions of the middle temporal visual area of the macaque monkey. J Neurosci 5:825-840.

Newsome WT, Mikami A, Wurtz RT (1986) Motion selectivity in macaque visual cortex. III. Psychophysics and physiology of apparent motion. J Neurophysiol 55:1340-1351.

Newsome WT, Wurtz RH, Komatsu H (1988) Relation of cortical areas MT and MST to pursuit eye movements. II. Differentiation of retinal from extraretinal inputs. J Neurophysiol 60:604-620.

Pasternak T, Merigan WH (1994) Motion perception following lesions in the superior temporal sulcus in the monkey. Cereb Cortex 4:247-259.

Pelli DG (1985) Uncertainty effects explain many aspects of visual contrast detection and discrimination. J Opt Soc Am A 2:1508-1531.

Plant GT, Nakayama K (1993) The characteristics of residual motion perception in the hemifield contralateral to lateral occipital lesions in humans. Brain 116:1337-1353.

Plant GT, Laxer KD, Barbaro NM, Schiffman JS, Nakayama K (1993) Impaired visual motion perception in the contralateral hemifield following unilateral posterior cerebral lesions in humans. Brain 116:1303-1335.

Rodman HR, Albright TD (1987) Coding of visual stimulus velocity in area MT of the macaque. Vision Res 27:2035-2048.

Rudolph KK, Pasternak T (1996) Motion and form perception after lesions of areas MT/MST and V4 in the macaque. Invest Ophthal Vis Sci 37:486.

Sary G, Vogels R, Orban GA (1993) Cue-invariant shape selectivity of macaque inferior temporal neurons. Science 260:995-997.
Sary G, Vogels R, Kovacs G, Orban GA (1995) Responses of monkey inferior temporal neurons to luminance-, motion-, and texture-defined gratings. J Neurophysiol 73:1341-1354.

Seeger W (1978) Atlas of topographical anatomy of the brain and surrounding structures. New York: Springer.

Shipp S, de Jong BM, Zihl J, Frackowiak RSJ, Zeki S (1994) The brain activity related to residual motion vision in a patient with bilateral lesions in V5. Brain 117:1023-1038.

Smith AT (1987) Velocity perception and discrimination: relation to temporal mechanisms. Vision Res 27:1491-1500.

Smith AT (1994) The detection of second-order motion. In: Visual detection of motion (Smith AT, Snowden RD, eds). New York: Academic.

Smith AT, Ledgeway T (1997) Separate detection of moving luminance and contrast modulations: fact or artifact? Vision Res 37:45-62.

Snowden RJ, Treue S, Andersen RA (1992) The response of neurons in areas V1 and MT of the alert rhesus monkey to moving random dot patterns. Exp Brain Res 88:389-400.

Thomas JP (1983) Underlying psychometric functions for detecting gratings and identifying spatial frequency. J Opt Soc Am A 73:751-758.

Thomas JP (1985) Detection and identification: how are they related? J Opt Soc Am A 2:1457-1467.

Tootell RBH, Reppas JB, Kwong KK, Malach R, Born RT, Brady TJ, Rosen BR, Belliveau JW (1995) Functional analysis of human MT and related visual cortical areas using magnetic resonance imaging. J Neurosci 15:3215-3230.

Vaina LM (1989) Selective impairment of visual motion interpretation following lesions of the right occipito-parietal area in humans. Biol Cybern 61:347-359.

Vaina LM, Grzywacz NM, Kikinis R (1994) Segregation of computations underlying the perception of motion discontinuity and coherence. NeuroReport 5:2289-2294.

Watson JDG, Myers R, Frackowiak RSJ, Hajnal JV, Woods RP, Mazziotta JC, Shipp S, Zeki S (1993) Area V5 of the human brain: evidence from a combined study using positron emission tomography and magnetic resonance imaging. Cereb Cortex 3:79-84.

Wilson HR, Ferrera VP, Yo C (1992) A psychophysically motivated model for two-dimensional motion perception. Visual Neurosci 9:79-97.

Zeki S, Watson JDG, Lueck CJ, Friston KJ, Kennard C, Frackowiak RSJ (1991) A direct demonstration of functional specialization in human visual cortex. J Neurosci 11:641-649.

Zihl J, von Cramon D, Mai N (1983) Selective disturbance of movement vision after bilateral brain damage. Brain 106:313-340.

Zihl J, von Cramon D, Mai N, Schmid C (1991) Disturbance of movement vision after bilateral posterior brain damage: further evidence and follow-up observations. Brain 114:2235-2252. 